\title{
Second Korean guidelines for the management of Crohn's disease
}

\author{
Jae Jun Park ${ }^{1}$, Suk-Kyun Yang ${ }^{2}$, Byong Duk Ye ${ }^{2}$, Jong Wook Kim³, Dong Il Park', \\ Hyuk Yoon ${ }^{5}$, Jong Pil $\operatorname{Im}^{6}$, Kang Moon Lee ${ }^{7}$, Sang Nam Yoon ${ }^{8}$, Heeyoung Lee; \\ IBD Study Group of the Korean Association for the Study of Intestinal Diseases \\ ${ }^{1}$ Department of Internal Medicine, Yonsei University College of Medicine, Seoul, ${ }^{2}$ Department of Internal Medicine, University of Ulsan College \\ of Medicine, Seoul, ${ }^{3}$ Department of Internal Medicine, Inje University College of Medicine Ilsan Paik Hospital, Goyang, ${ }^{4}$ Department of Internal \\ Medicine, Sungkyunkwan University School of Medicine, Seoul, ${ }^{5}$ Department of Internal Medicine, Seoul National University Bundang \\ Hospital, Seongnam, ${ }^{6}$ Department of Internal Medicine, Seoul National University Hospital, Seoul, ${ }^{7}$ Department of Internal Medicine, The \\ Catholic University of Korea College of Medicine, Suwon, ${ }^{8}$ Department of Surgery, Hallym University College of Medicine, Chuncheon, ${ }^{9}$ Center \\ for Preventive Medicine and Public Health, Seoul National University Bundang Hospital, Seongnam, Korea
}

Crohn's disease (CD) is a chronic, progressive, and disabling inflammatory bowel disease (IBD) with an uncertain etiopathogenesis. CD can involve any site of the gastrointestinal tract from the mouth to the anus, and is associated with serious complications, such as bowel strictures, perforations, and fistula formation. The incidence and prevalence rates of CD in Korea are still lower compared with those in Western countries, but they have been rapidly increasing during the recent decades. Although there are no definitive curative modalities for $\mathrm{CD}$, various medical and surgical therapies have been applied for the treatment of this disease. Concerning CD management, there have been substantial discrepancies among clinicians according to their personal experience and preference. To suggest recommendable approaches to the diverse problems of CD and to minimize the variations in treatment among physicians, guidelines for the management of CD were first published in 2012 by the IBD Study Group of the Korean Association for the Study of Intestinal Diseases. These are the revised guidelines based on updated evidence, accumulated since 2012. These guidelines were developed by using mainly adaptation methods, and encompass induction and maintenance treatment of CD, treatment based on disease location, treatment of CD complications, including stricture and fistula, surgical treatment, and prevention of postoperative recurrence. These are the second Korean guidelines for the management of CD and will be continuously revised as new evidence is collected. (Intest Res 2017;15:38-67)

Key Words: Crohn disease; Management; Guideline

Received January 13, 2017. Revised January 15, 2017

Accepted January 16, 2017.

Correspondence to Suk-Kyun Yang, Department of Gastroenterology, Asan

Medical Center, University of Ulsan College of Medicine, 88 Olympic-ro, 43-

gil, Songpa-gu, Seoul 05505, Korea. Tel: +82-2-3010-3901, Fax: +82-2-

476-0824,E-mail: sky@amc.seoul.kr

These guidelines are being co-published on the Korean Journal of Gastroenterology and the Intestinal Research for facilitated distribution.

Financial support: This work was supported by the Research Program funded by the Korea Centers for Disease Control and Prevention (2016E63001-00). Conflict of interest: None.

\section{INTRODUCTION}

Crohn's disease (CD) is a chronic intractable inflammatory bowel disease (IBD) that can involve any part of the gastrointestinal tract from the mouth to the anus. The main symptoms of CD are abdominal pain, diarrhea, and weight loss. It occurs mainly in the 10 to 20 years age group and lasts throughout a lifetime, as well as causes complications such as bowel stenosis, fistula, and perforation. The etiology of CD is still unclear; however, it is presumed to be a combination of genetic factors, environmental factors, and abnormal immune responses to intestinal microflora. CD is

\footnotetext{
๑ Copyright 2017. Korean Association for the Study of Intestinal Diseases. All rights reserved.

This is an Open Access article distributed under the terms of the Creative Commons Attribution Non-Commercial License (http://creativecommons.org/licenses/by-nc/4.0)

which permits unrestricted non-commercial use, distribution, and reproduction in any medium, provided the original work is properly cited.
} 
a common disease in developed Western countries but is a very rare disease in Asia including Korea. However, recent epidemiological studies have shown that the incidence of CD in East Asian countries including Korea is continuously increasing, ${ }^{1-3}$ therefore, the social and economic burdens caused by $\mathrm{CD}$ are also gradually increasing.

Although CD is still being considered an incurable disease, various therapies have been attempted for the various clinical manifestations and complications of this disease. Recently, many treatment policies based on the results of clinical studies have been proposed. However, much of the treatment of CD is still based on the experience and judgment of the physician, and there are many variations in the treatment methods among clinicians. To reduce these discrepancies and to suggest appropriate, evidence-based management in each clinical situation, the IBD Study Group of the Korean Association for the Study of Intestinal Diseases (KASID) in 2012 developed guidelines for the management of CD in Korea based on North American and European guidelines. ${ }^{4,5}$ Since then, many studies have been published, new knowledge has been accumulated, and treatment guidelines based on the latest evidence have been proposed. Accordingly, the revision committee has developed a revised edition of the guidelines for the management of $\mathrm{CD}$ based on the eight new guidelines ${ }^{6-13}$ published in the last 5 years.

These treatment guidelines cover mainly medical and surgical treatments (indications for surgery) of adult patients with CD. Meanwhile, treatments in special situations such as extraintestinal manifestations, pediatric cases, pregnancy, and lactation, vaccination, diet, and surveillance of colon cancer were excluded.

These guidelines for the management of $\mathrm{CD}$ are not intended to be an absolute therapeutic standard; however, based on scientific evidence to date, they have been developed with the aim of helping physicians in making decisions in the treatment of CD. Therefore, the medical practice for individual patients should be decided by the physician in charge, considering the various situations of the patient. These management guidelines should not be used for the purpose of restricting the practitioner's medical practice or as the standard for health insurance review, nor for making legal judgments about the medical practice performed for a specific patient. We hope that these management guidelines will help in resolving problems such as overtreatment, inadequate treatment, and delayed treatment of $\mathrm{CD}$, and in facilitating communication among clinicians and researchers by encouraging the use of uniform terminology for the disease.

\section{METHODS}

\section{Planning}

The IBD Study Group of KASID decided to develop a revised edition of the guidelines for the management of CD in July 2015 , owing to the need to revise the previous guidelines ${ }^{4,5}$ published in 2012.

The guideline development committee was administered by the IBD study group of KASID, and the subcommittee on the development of the guidelines for the management of CD was composed of eight gastroenterologist of IBD Study Group of KASID (Jae Jun Park, Jong Wook Kim, Dong Il Park, Kang Moon Lee, Jong Pil Im, Byong Duk Ye, Hyuk Yoon, and Suk-Kyun Yang), and one surgeon of The Korean Society of Coloproctology (Sang Nam Yoon). The first meeting was held on July 2, 2015, with a specialist on methodology of guidelines development (Heeyoung Lee) to discuss the revision direction of the guidelines for the management of CD.

A decision was made to revise the guidelines for gastroenterologists by covering mainly medical and surgical treatment (indications for surgery) of adult patients with CD, and the detailed contents include remission induction and maintenance therapy of CD, treatment based on disease location, treatment for stricturing or fistulizing disease, surgical treatment, and prevention of postoperative recurrence. Meanwhile, treatments in special situations including extraintestinal manifestations, pediatric patients, pregnancy, and lactation, vaccination, diet, and surveillance of colon cancer were excluded.

\section{Process of Development}

\section{1) Selection of the Key Questions}

After the meeting and discussion of the subcommittee on guideline development, key questions were selected on the basis of the questions raised in the clinical field. It was decided that one of these key questions will be developed by using a de novo method and the remaining key questions will be developed with an adaptation method.

\section{2) Searching for Source Guidelines}

A meticulous search for medical guidelines from January 2010 to June 2015, by using the portal site for medical guidelines and major domestic and foreign databases, was conducted; thereafter, 57 documents were extracted. 


\section{3) Assessment of Guideline Qualities and Selection of Guidelines}

Among the searched guidelines for the management of $\mathrm{CD}$, guidelines that were developed by using an evidencedbased method, with national or international level, and underwent a peer-review process were first selected. For each document, two committee members evaluated the fidelity of the contents and the clinical applicability, by using the clinical guideline assessment tools of Appraisal of Guidelines Research and Evaluation II. Finally, eight guidelines ${ }^{6-13}$ that fully satisfied the selection criteria were selected (Table 1).

\section{4) Adaptation}

Concerning the predefined key questions, the evidences and recommendations of eight selected guidelines were reviewed, scrutinized, and summarized. Thereafter, recommendations for predefined key questions were drawn, and the background evidence was described. The quality of evidence and the classification of recommendation in these guidelines are presented according to the Grading of Recommendation Assessment, Development and Evalua- tion (GRADE) format. ${ }^{14-16}$ Following the GRADE format, the quality of evidence for each recommendation is assessed as high, moderate, low, or very low. The strength of recommendation is classified as strong or weak based on four main components: desirable and undesirable effects, quality of the evidence, values and preference, and resource allocation. ${ }^{16,17}$ The definition of quality of evidence and classification of recommendation is described in Table 2. ${ }^{14-17}$

\section{5) De novo Development}

De novo development was conducted for one following key question according to the GRADE format: the efficacy of anti-tumor necrosis factor (anti-TNF) for the prevention of postoperative recurrence of CD. PubMed and EMBASE were used for searching evidences about the key question (Supplementary Table 1). Concerning the inclusion criteria, language was limited to Korean and English, and the study design included randomized controlled trials (RCTs) and nonrandomized comparative studies. Editorials, letters, and proceedings were excluded. The final analysis was conducted for the selected studies ${ }^{18-24}$ that met all of the inclusion

Table 1. Eight Guidelines Selected with AGREE II Instrument

\begin{tabular}{|c|c|c|c|c|c|}
\hline No. & Title & $\begin{array}{l}\text { Country/ } \\
\text { language }\end{array}$ & Journal & Year & Volume/page \\
\hline 1 & $\begin{array}{l}\text { The London Position Statement of the World Congress of } \\
\text { Gastroenterology on Biological Therapy for IBD With the European } \\
\text { Crohn's and Colitis Organization: when to start, when to stop, } \\
\text { which drug to choose, and how to predict response? }\end{array}$ & $\begin{array}{l}\text { United Kingdom/ } \\
\text { English }\end{array}$ & $\begin{array}{l}\text { American Journal of } \\
\text { Gastroenterology }\end{array}$ & 2011 & 106/199-212 \\
\hline 2 & $\begin{array}{l}\text { The Italian Society of Gastroenterology (SIGE) and the Italian Group for } \\
\text { the Study of Inflammatory Bowel Disease (IG-IBD) Clinical Practice } \\
\text { Guidelines: the use of TNF- } \alpha \text { antagonist therapy in inflammatory } \\
\text { bowel disease }\end{array}$ & Italy/English & $\begin{array}{l}\text { Digestive and Liver } \\
\text { Disease }\end{array}$ & 2011 & $43 / 1-20$ \\
\hline 3 & $\begin{array}{l}\text { Guidelines for the management of inflammatory bowel disease in } \\
\text { adults }^{8}\end{array}$ & $\begin{array}{l}\text { United Kingdom/ } \\
\text { English }\end{array}$ & Gut & 2011 & $60 / 571-607$ \\
\hline 4 & Crohn's disease: management in adults, children and young people ${ }^{a, 9}$ & $\begin{array}{l}\text { United Kingdom/ } \\
\text { English }\end{array}$ & NA & 2012 & $N A / 1-398$ \\
\hline 6 & $\begin{array}{l}\text { Evidence-based clinical practice guidelines for Crohn's disease, } \\
\text { integrated with formal consensus of experts in Japan }{ }^{11}\end{array}$ & Japan/English & $\begin{array}{l}\text { Journal of } \\
\text { Gastroenterology }\end{array}$ & 2013 & $48 / 31-72$ \\
\hline 7 & $\begin{array}{l}\text { A global consensus on the classification, diagnosis and } \\
\text { multidisciplinary treatment of perianal fistulising Crohn's disease }\end{array}$ & $\begin{array}{l}\text { The Netherlands/ } \\
\text { English }\end{array}$ & Gut & 2014 & 63/1381-1392 \\
\hline 8 & $\begin{array}{l}\text { Asia-Pacific consensus statements on Crohn's disease. Part 2: } \\
\text { management }{ }^{13}\end{array}$ & Australia/English & $\begin{array}{l}\text { Journal of } \\
\text { Gastroenterology } \\
\text { and Hepatology }\end{array}$ & 2016 & $31 / 56-68$ \\
\hline
\end{tabular}

${ }^{a}$ Guidelines are freely available on the web (https://www.ncbi.nlm.nih.gov/pubmedhealth/PMH0068978/).

NA, not applicable; anti-TNF, anti-tumor necrosis factor. 
Table 2. Definitions or Implications of the Levels of Evidence and Recommendations

\begin{tabular}{|c|c|}
\hline Level & Definition/implication \\
\hline High & We are very confident that the true effect lies close to that of the estimate of the effect. \\
\hline Low & $\begin{array}{l}\text { Our confidence in the effect estimate is limited: the true effect may be substantially different from the } \\
\text { estimate of the effect. }\end{array}$ \\
\hline Very low & $\begin{array}{l}\text { We have very little confidence in the effect estimate: the true effect is most likely to be substantially different } \\
\text { from the estimate of the effect. }\end{array}$ \\
\hline \multicolumn{2}{|c|}{ Classification of recommendations } \\
\hline Strong & Most patients should receive the recommended course of action. \\
\hline Weak & $\begin{array}{l}\text { Clinicians should recognize that different choices would be appropriate for different patients and that they } \\
\text { must help patients to arrive at a management decision consistent with his or her values and preferences. }\end{array}$ \\
\hline
\end{tabular}

criteria (Supplementary Table 2, Supplementary Fig. 1). On the basis of the derived results, the recommendation was confirmed, and the quality of evidence and classification of recommendation were given.

\section{6) Delphi Process for the Agreement to Recommendations}

On September 25, 2016, the draft of the updated Korean guidelines for the management of CD was presented during the consensus meeting that was attended by 53 IBD specialists from Korea. Each recommendation of the guidelines was classified into five levels: "strongly agree," "agree," "uncertain," "disagree," or "strongly disagree." Each recommendation of the guidelines was accepted when more than $75 \%$ of the participants had chosen "strongly agree" or "agree." For recommendations with below $75 \%$ agreement, the guideline subcommittee again conducted a discussion and made modifications. Thereafter, the revised recommendation underwent a second voting via the Internet. The secondary voting for the guidelines was participated in by 46 IBD specialists out of the 53 participants of the primary evaluation. The degree of final agreement was indicated as a percentage value below the evidence level, assigned as "level of agreement."

\section{7) Internal Review, Endorsement, and Distribution of Guidelines}

The revised draft was reviewed and approved by the members of KASID. The final draft is being co-published by the Korean Journal of Gastroenterology and the Intestinal Research for facilitated distribution, and will be distributed by the Korean Medical Guideline Information Center (http:// www.guideline.or.kr). Moreover, following distribution, the revised edition is intended for later publication based on user opinions and the newly published literature about the management of CD.

\section{THERAPEUTIC APPROACH}

The goal of the treatment of CD is to induce and maintain a remission of active disease, ultimately improving the health and quality of life of the patient. ${ }^{25,26}$ Moreover, prevention of complications by precluding the progression of disease has emerged as an important therapeutic goal. ${ }^{27}$

Several cohort studies ${ }^{28-32}$ that analyzed the long-term clinical outcomes of CD have shown that although treatment with the primary goal of loss of symptoms (clinical remission) is performed, bowel damage progresses resulting in subsequent operation owing to the occurrence of complications in a substantial number of patients; thus, the concern that a symptoms-based treatment strategy cannot improve the natural course of CD has been raised. Therefore, a therapeutic approach that can prevent the progression of CD through the adequate use of drugs, such as immunomodulators or biological agents with a potential to induce healing of inflamed bowel before the occurrence of irreversible bowel damage, is increasingly being applied in clinical practice. However, because the use of these drugs is accompanied with concerns of drug adverse effects and high costs, this treatment approach may be preferentially considered for patients who are expected to have a poor prognosis. The factors associated with poor prognosis of CD include young age at diagnosis, involvement of both the ileum and colon, presence of perianal lesions, and use of steroids at diagnosis. ${ }^{33,34}$

In the treatment of $\mathrm{CD}$, the activity of the disease, site of involvement (ileum, ileum and colon, colon, or other site), and 
disease behavior (inflammatory, structuring, and fistulizing) should always be taken into account. ${ }^{35}$ Moreover, the drug formulation and action site, drug efficacy and adverse effects, response to previous treatments (relapse, steroid dependency, steroid refractoriness, etc.), extraintestinal involvement, and complications should also be considered. ${ }^{35}$ When the disease is judged to be in the active phase, in addition to CD activity, the possibility of intestinal infections, abscesses, fibrotic stricture, bacterial overgrowth, malabsorption of bile salts, abnormal bowel motility, and gallstone disease must also be ruled out. ${ }^{35}$ Furthermore, the treatment decision should be individualized according to the patient's state, and should be made after sufficient discussion with the patient.

\section{Disease Activity of CD}

\section{1) Disease Activity Index}

The index of clinical disease activity of $\mathrm{CD}$ is represented by the $\mathrm{CDAI}^{36}$ (Table 3) and the Harvey-Bradshaw index (HBI) ${ }^{37}$ (Table 4). CDAI is widely used in many clinical studies and clinical fields. A CDAI of $<150$ is classified as remission, 150 to $<220$ as mild activity, 220 to $<450$ as moderate activity, and $\geq 450$ as severe activity. ${ }^{38}$ On the other hand, HBI is a disease activity index designed to simplify the complex CDAI, and consists of only clinical parameters. An HBI of $<5$ is classified as remission, 5 to $<8$ as mild activity, 8 to $<16$ as moderate activity, and $\geq 16$ as severe activity. ${ }^{37,39}$

\section{2) Definition of Terminology}

Response is generally defined as a reduction in CDAI of

Table 3. Crohn's Disease Activity Index

\begin{tabular}{|c|c|c|c|c|}
\hline No. & Item & Description & & Multiplier \\
\hline 1 & $\begin{array}{l}\text { Number of liquid or very soft } \\
\text { stools }\end{array}$ & Sum of 7 day & - & $\times 2$ \\
\hline 2 & Abdominal pain & Sum of 7 day & 0 , none; 1 , mild; 2 , moderate; 3 , severe & $\times 5$ \\
\hline 3 & General well-being & Sum of 7 day & $\begin{array}{l}0, \text { generally well; } 1 \text {, slightly under par; } 2 \text {, poor; } \\
\text { 3, very poor; 4, terrible }\end{array}$ & $\times 7$ \\
\hline 4 & $\begin{array}{l}\text { Number of six listed categories } \\
\text { patient now has }\end{array}$ & $\begin{array}{l}\text { Number of six listed } \\
\text { categories }\end{array}$ & $\begin{array}{l}\text { (1) Arthritis/arthralgia } \\
\text { (2) Iritis/uveitis } \\
\text { (3) Erythema nodosum/pyoderma } \\
\text { gangrenosum/aphthous stomatitis } \\
\text { (4) Anal fissure, fistula, or abscess } \\
\text { (5) Other fistula } \\
\text { (6) Fever }>37.8^{\circ} \mathrm{C}\left(100^{\circ} \mathrm{F}\right) \text { during the past week }\end{array}$ & $\times 20$ \\
\hline 5 & Antidiarrheal drug use & Use in the previous 7 day & 0, no; 1 , yes & $\times 30$ \\
\hline 6 & Abdominal mass & - & 0 , none; 2 , questionable; 5 , definite & $\times 10$ \\
\hline 7 & Hematocrit & $\begin{array}{l}\text { Expected-observed } \\
\text { Hematocrit }\end{array}$ & $\begin{array}{l}\text { Male, 47-hematocrit } \\
\text { Female, 42-hematocrit }\end{array}$ & $\times 6$ \\
\hline 8 & Body weight & \multicolumn{2}{|c|}{ Percent below standard weight (normogram) } & $\times 1$ \\
\hline
\end{tabular}

Table 4. Harvey-Bradshaw Simple Index

\begin{tabular}{lll}
\hline Variable & \multicolumn{1}{c}{ Description } & \multicolumn{1}{c}{ Scoring } \\
\hline 1 & General well-being & 0, very well; 1, slightly below par; 2, poor; 3, very poor; 4, terrible \\
2 & Abdominal pain & 0, none; 1, mild; 2, moderate; 3, severe \\
3 & Number of liquid stools daily & 1 Per occurrence \\
4 & Abdominal mass & 0, none; 1, dubious; 2, definite; 3, definite and tender \\
5 & Complications & 1 Per item: arthralgia, uveitis, erythema nodosum, aphthous ulcer, pyoderma gangrenosum, \\
& & anal fissure, new fistula, abscess \\
Total score & & Sum of variable scores \\
\hline
\end{tabular}


$\geq 100 .{ }^{38}$ In some studies, a reduction of $\geq 70$ is defined as a response. ${ }^{40,41}$ Meanwhile, for HBI, response is defined as a reduction of $\geq 3$. ${ }^{42}$

Relapse is defined as the recurrence of a symptom in a patient with clinical remission, and is generally defined as CDAI $>150 .{ }^{38}$ In some clinical studies, relapse was defined as CDAI $>150$ plus an increase of 70 or 100 , which somewhat differed among those studies. ${ }^{38,39}$ Meanwhile, for HBI, relapse is defined as HBI $>4 .^{38}$ Early relapse can be defined as the recurrence of a symptom within 3 months after remission. ${ }^{43}$

Steroid-refractory disease is defined as sustained activity even after prednisolone therapy at a dose of $0.75 \mathrm{mg} / \mathrm{kg} / \mathrm{day}$ during 4 weeks. ${ }^{35}$ Steroid dependency is defined as the inability to taper prednisolone to $<10 \mathrm{mg}$ /day (budesonide $3 \mathrm{mg} /$ day) owing to disease activity within 3 months of starting steroids, or relapse within 3 months after steroid discontinuation. ${ }^{35}$

\section{3) Other Activity Assessment Methods}

In addition to CDAI or HBI, it is recommended that objective tests for disease activity, including blood inflammatory markers such as C-reactive protein (CRP), fecal inflammatory markers such as calprotectin, or colonoscopy, be performed, if available, before starting or modifying treatment. ${ }^{35}$

\section{Classification of CD}

The Vienna classification system, ${ }^{44}$ proposed in 1998, which is based on the age at diagnosis (A1, <40 years; A2, $\geq 40$ years), involved site (L1, ileum; L2, colon; L3, ileum

Table 5. Montreal Classification for CD

\begin{tabular}{|c|c|}
\hline Variable & \\
\hline \multirow[t]{3}{*}{ Age at diagnosis (yr) } & $A 1, \leq 16$ \\
\hline & $A 2,17-39$ \\
\hline & $A 3, \geq 40$ \\
\hline \multirow[t]{4}{*}{ Location } & L1, ileal \\
\hline & L2, colonic \\
\hline & L3, ileocolonic \\
\hline & L4, isolated upper disease ${ }^{a}$ \\
\hline \multirow[t]{4}{*}{ Behavior } & B1, non-stricturing, non-penetrating \\
\hline & B2, stricturing \\
\hline & B3, penetrating \\
\hline & p, perianal disease modifier ${ }^{b}$ \\
\hline
\end{tabular}

and colon; L4, upper gastrointestinal tract), and disease behavior (B1, inflammatory [nonstricturing, nonpenetrating]; B2, stricturing; B3, fistulizing [penetrating]), was widely used to classify CD in the past; however, the Montreal classification $^{45}$ (Table 5) proposed in 2005 is now used as the standard classification method for CD in adult patients. The Montreal classification was proposed through modification and supplementation of the Vienna classification. Because younger age at diagnosis was known to be highly related to genetic mutation, the age of 40 years was subdivided again into $\leq 16$ years or $>16$ years in the Montreal classification. As for the involved site, upper gastrointestinal disease (L4) can be added to L1-L3, whereas for disease behavior, the presence of perianal fistula or perianal abscess (p) can be added to B1-B3. In adult CD, it has been known that the site of disease involvement generally does not change over time, but disease behavior is known to progress from the inflammatory to the stricturing or fistulizing type in a substantial number of patients. ${ }^{46,47}$

CD can also be classified according to the degree of inflammatory lesion involvement in the gastrointestinal tract. Localized disease is defined as intestinal involvement $<30$ $\mathrm{cm}$ in length regardless of the anatomical location. This is usually the case when the ileocecal area is involved, and the sum of the length of the involved ileum and ascending colon is the criterion. ${ }^{38}$ Meanwhile, extensive disease is defined as a total length of involvement of $\geq 100 \mathrm{~cm}$ regardless of the anatomical location. ${ }^{38}$

\section{INDUCTION THERAPY FOR CD}

\section{Mild to Moderate CD}

1. Sulfasalazine can be used to induce remission of mild colonic CD (quality of evidence, high; classification of recommendation, strong).

- Level of agreement: strongly agree 24\%, agree 67\%, uncertain 7\%, disagree 2\%, strongly disagree $0 \%$

2. Although the efficacy of 5-aminosalicylic acid (5-ASA) for the remission induction of mild CD is limited, the use of this drug may be considered because of its fewer adverse effects and ease of administration (quality of evidence, high; classification of recommendation, weak).

- Level of agreement: strongly agree 20\%, agree 72\%, uncertain $8 \%$, disagree $0 \%$, strongly disagree $0 \%$

3. Systemic corticosteroids are indicated for mild active $C D$ that is refractory to 5-ASA (quality of evidence, high; classification of recommendation, strong).

- Level of agreement: strongly agree 36\%, agree 53\%, uncertain $9 \%$, disagree $0 \%$, strongly disagree $2 \%$ 
4. Budesonide (9 $\mathrm{mg} / \mathrm{day})$ is preferred for induction therapy of mild to moderate CD confined to the terminal ileum or ileocecal area (quality of evidence, high; classification of recommendation, strong).

- Level of agreement: strongly agree 9\%, agree 87\%, uncertain $4 \%$, disagree $0 \%$, strongly disagree $0 \%$

5. Systemic corticosteroid should be used if budesonide is not effective (quality of evidence, high; classification of recommendation, strong).

- Level of agreement: strongly agree 38\%, agree 58\%, uncertain 4\%, disagree 0\%, strongly disagree 0\%

In a meta-analysis of RCTs, sulfasalazine doses of 3 to $6 \mathrm{~g} /$ day were superior to placebo in the induction therapy of mild to moderate active CD (pooled risk ratio, 1.38), ${ }^{48}$ and efficacy was seen only in patients with colonic involvement but not in patients with disease limited to the small intestine. ${ }^{49-51}$

In several placebo-controlled trials conducted in the 1990s, it was reported that mesalamine, a 5-ASA, is efficacious for mild ileocecal CD. ${ }^{52,53}$ Because of not only these evidences but also its fewer adverse effects and ease of administration, mesalamine has been widely used in the treatment of CD. Meanwhile, in a meta-analysis of three largescale trials that evaluated the efficacy of $4 \mathrm{~g} /$ day mesalamine for active CD, high-dose mesalamine showed a statistically significant difference in the reduction of the CDAI in patients with active CD compared with placebo $(P=0.04)^{54}$; however, the difference in the mean value of decreased CDAI (mesalamine group, -63 ; placebo group, -45 ; difference of CDAI value between the two groups, 18) was small between the two groups, and its clinical usefulness is limited.

Budesonide controlled ileal release capsules are used for the treatment of patients with CD mainly involving the ileocecal area, because the drug starts to be released at pH 5.5 or higher and is mostly released and exert actions in the terminal ileum and proximal colon.$^{55}$ In the Cochrane review, enteric-coated budesonide had a significantly higher remission-inducing efficacy within 8 weeks than placebo (RR, 1.96; 95\% CI, 1.19-3.23) and mesalamine (RR, 1.63; 95\% CI, 1.232.16) in mild to moderate active $\mathrm{CD}{ }^{56}$ Budesonide has fewer adverse effects than conventional systemic corticosteroids (RR, 0.64; 95\% CI, 0.54-0.76), as it undergoes a high firstpass metabolism in the pharmacokinetics of the body.$^{56}$ The recommended dose of budesonide is $9 \mathrm{mg} /$ day orally, and is usually reduced by 3 mg every 2 to 4 weeks after 6 weeks of administration. Meanwhile, in patients with high disease activity, systemic corticosteroids are more effective in inducing remission than budesonide. According to the analysis of two individual studies, the pooled risk ratio of remission induc- tion was 0.52 (95\% CI, 0.28-0.95), indicating that systemic corticosteroids were superior to budesonide. ${ }^{57,58}$ Therefore, budesonide is preferred in patients with mild to moderate CD localized to the terminal ileum or ileocecal area, whereas systemic corticosteroids are recommended for patients with higher disease activity. If sufficient efficacy is not achieved even after administration of budesonide for an adequate period, the use of systemic corticosteroids is considered.

\section{Moderate to Severe CD}

6. Systemic corticosteroid (prednisolone 0.5 to $1 \mathrm{mg} /$ $\mathrm{kg} /$ day or 40 to $60 \mathrm{mg} /$ day) is the first-line induction therapy for moderate to severe CD (quality of evidence, moderate; classification of recommendation, strong).

- Level of agreement: strongly agree 38\%, agree 60\%, uncertain 2\%, disagree 0\%, strongly disagree 0\%

7. Systemic corticosteroid should be reduced gradually according to disease severity and patient response, generally over $\mathbf{8}$ weeks (quality of evidence, low; classification of recommendation, strong).

- Level of agreement: strongly agree 39\%, agree $61 \%$, uncertain $0 \%$, disagree $0 \%$, strongly disagree $0 \%$

8. Anti-TNF therapy is indicated if systemic corticosteroid therapy fails (quality of evidence, high; classification of recommendation, strong).

- Level of agreement: strongly agree $48 \%$, agree $48 \%$, uncertain $4 \%$, disagree $0 \%$, strongly disagree $0 \%$

9. Thiopurine monotherapy is not recommended for induction therapy of moderate to severe CD (quality of evidence, moderate; classification of recommendation, weak).

- Level of agreement: strongly agree 19\%, agree 65\%, uncertain $14 \%$, disagree $2 \%$, strongly disagree $0 \%$

10. Anti-TNF agents may be used to induce remission of moderate to severe CD (quality of evidence, moderate; classification of recommendation, weak).

- Level of agreement: strongly agree 37\%, agree 63\%, uncertain $0 \%$, disagree $0 \%$, strongly disagree $0 \%$

11. When anti-TNF is used for induction therapy of thiopurine-naïve patients, combined therapy with anti-TNF and thiopurine is more effective than anti-TNF alone (quality of evidence, moderate; classification of recommendation, weak).

- Level of agreement: strongly agree $34 \%$, agree $62 \%$, uncertain $2 \%$, disagree $2 \%$, strongly disagree $0 \%$

12. Intramuscular methotrexate (MTX) may be used to induce remission for moderate to severe CD (quality of evidence, high; classification of recommendation, weak).

- Level of agreement: strongly agree $11 \%$, agree $85 \%$, uncertain $4 \%$, disagree $0 \%$, strongly disagree $0 \%$ 
According to the Cochrane reviews of RCTs that tested the efficacy of systemic corticosteroid therapy in active CD, systemic corticosteroids showed a significantly higher efficacy of remission induction than did placebo (RR, 1.99; 95\% CI, 1.51-2.64). ${ }^{59}$ Looking at the individual studies included in this Cochrane review, Summers et al. ${ }^{60}$ randomly assigned 162 patients with active $\mathrm{CD}$ to either prednisone (0.5-0.75 $\mathrm{mg} / \mathrm{kg} /$ day, gradual reduction) or placebo, and remission was induced in $30 \%$ of the control group but in $60 \%$ of the corticosteroid group at 17 weeks (number needed to treat [NNT], 3). ${ }^{60}$ Meanwhile, in a study conducted by Malchow et al. ${ }^{49}$ (including 105 patients with active CD), remission was induced in $38 \%$ of the control group and in $83 \%$ of the 6-methylprednisolone ( $48 \mathrm{mg} /$ day, dose tapered every week) group during an 18-week period (NNT, 2).

To date, there has been no dose-response study for prednisolone. Prednisolone 0.5 to $1 \mathrm{mg} / \mathrm{kg} / \mathrm{day}$ or 40 to $60 \mathrm{mg} /$ day is usually recommended for induction therapy of moderate to severe CD. Moreover, there have been no studies on the effect of the route of corticosteroid administration, which is generally determined according to the severity of the disease of the patient. Intravenous corticosteroid therapy is considered in severe patients requiring hospitalization, whereas in other cases, corticosteroids are administered orally. Assessment of the therapeutic response to corticosteroids is generally done within 2 to 4 weeks for oral administration and within 1 to 2 weeks for intravenous administration. After the administration of systemic corticosteroids, the treatment response is assessed according to the severity of the patient, and if it is determined that sufficient therapeutic response has been achieved, the corticosteroid dose should be reduced. As rapid reduction is associated with early relapse, gradual tapering of corticosteroids over 8 weeks is recommended. ${ }^{8}$ Although systemic corticosteroids are highly effective in inducing remission of active CD, they are not effective in maintaining remission and can cause many adverse effects during long-term use. ${ }^{61}$

Infliximab is a chimeric IgG1 anti-TNF monoclonal antibody that was the first anti-TNF agent tried for the treatment of CD. In a randomized controlled study on the efficacy of infliximab for remission induction, 108 patients with moderate to severe CD who did not respond to 5-ASA, steroids, or immunomodulators (59\% of patients at the start of the study used systemic corticosteroids) were randomly assigned to the infliximab $(5,10$, or, $20 \mathrm{mg} / \mathrm{kg})$ or placebo group. In this study, the response rates at week 4 in the $5,10,20 \mathrm{mg} / \mathrm{kg}$ infliximab groups were $81 \%, 50 \%$, and $64 \%$, respectively, and the infliximab groups (overall response rate, $65 \%$ ) showed a higher response rate than the placebo group (overall response rate, $17 \%)(P<0.001){ }^{62}$ Moreover, the remission rate at 4 weeks was also significantly different between the infliximab (33\%) and placebo (4\%) groups $(P=0.005)$. Although the duration of the infliximab treatment response varied somewhat among patients, the response rate of the infliximab group at 12 weeks was $41 \%$, which was higher than that of the placebo group (12\%) $(P=0.008)$, and this confirms the persistence of drug efficacy. ${ }^{62}$

Adalimumab is a fully human anti-TNF agent administered subcutaneously. In the Clinical Assessment of Adalimumab Safety and Efficacy Studied as Induction Therapy in Crohn's Disease (CLASSIC)-I study, 299 patients with moderate to severe CD ( $20 \%$ of patients at the beginning of the study used systemic corticosteroids) who had not received prior infliximab were randomized to receive $40 / 20 \mathrm{mg}$ adalimumab, 80/40 mg adalimumab, 160/80 mg adalimumab, and placebo, respectively, at weeks 0 and 2 . The remission rates in the adalimumab groups at 4 weeks were $18 \%, 24 \%$, and $36 \%$, respectively, and the rate was significantly higher in the 160/80 mg group than in the placebo group (12\%) $(P=0.001) .{ }^{63}$ In the above infliximab and adalimumab studies, as the treatment results for patients who failed systemic corticosteroids therapy were not presented separately, the efficacy of anti-TNF agents in these patients are not known exactly. Certolizumab pegol (certolizumab), another anti-TNF agent, is a type of polyethylene glycol attached to anti-TNF Fab'. Unlike infliximab or adalimumab, certolizumab does not cause apoptosis of $\mathrm{T}$ cells and monocytes, and it did not consistently show a definite effect in the placebo-controlled studies of patients with moderate to severe $\mathrm{CD}^{64-66}$

Although thiopurine (azathioprine [AZA] or 6-mercaptopurine [6-MP]) had been reported to be effective in inducing remission of active $\mathrm{CD}$ in an earlier meta-analysis, ${ }^{67}$ the latest Cochrane review showed that thiopurine was not more effective than placebo in inducting remission for active luminal CD (RR, 1.23; 95\% CI, 0.97-1.55), and in improving or healing fistula in CD (RR, 2.00; 95\% CI, $0.67-5.93) .{ }^{68}$ Therefore, the use of thiopurine alone for the induction of moderate to severe CD lacks clinical evidence.

The Study of Biologic and Immunomodulator Naive Patients in Crohn's Disease (SONIC) was conducted by randomly assigning infliximab plus AZA, infliximab alone, or AZA alone to patients with moderate to severe CD who had never received AZA or infliximab. The primary end point of the study was the proportion of patients who reached a steroid-free remission at week 26 , which was significantly higher in the group with the combination therapy of inflix- 
imab and AZA than in the infliximab-alone group (56.8\% vs. $44.4 \%$; $P=0.02)$ or AZA-alone group $(56.8 \%$ vs. $30.0 \%$; $P<0.01) .{ }^{69}$ In addition, concerning the mucosal healing rate at 26 weeks, the combination therapy group showed a higher trend of mucosal healing rate than the infliximabalone group ( $43.9 \%$ vs. $30.1 \% ; P=0.06$ ), and demonstrated a significantly higher mucosal healing rate than the AZAalone group ( $43.9 \%$ vs. $16.50 \%$; $P<0.01) .{ }^{69}$ Meanwhile, according to the meta-analysis that evaluated the efficacy of immunomodulator combination therapy for adalimumab in $\mathrm{CD}$, adalimumab monotherapy was less effective than the combination therapy of adalimumab and immunomodulator in the remission induction of CD (OR, 0.78; 95\% CI, $0.64-0.96 ; P=0.02)$. However, there was no difference in the maintenance efficacy between the two groups (OR, 1.08; $95 \%$ CI, $0.79-1.48 ; P=0.48){ }^{70}$ The previous SONIC study was conducted on thiopurine-naïve patients; however, the individual studies included in the aforementioned meta-analysis of adalimumab were performed on patients regardless of the history of immunomodulatory use. Thus, caution is needed in the comparative interpretation of the results from those two studies.

MTX is an antimetabolite that competitively inhibits dihydrofolate reductase, and exerts its effect by blocking the action of dihydrofolate in the cells for DNA synthesis and cell division. ${ }^{71,72}$ However, the effect of MTX in inflammatory diseases such as rheumatoid arthritis is not explained by the cytotoxic mechanism, and it is presumed that its action through inhibition of interleukin or an eicosanoid compound may be involved in the suppression of inflammatory response. ${ }^{73}$ According to the placebo-controlled study on the efficacy of MTX in inducing remission in 141 patients with active CD, patients receiving intramuscular MTX $25 \mathrm{mg} /$ wk had significantly higher remission rates at week 16 than those who received placebo (39\% vs. $19 \% ; P=0.025$; NNT, 5$).^{74}$ On the other hand, in placebo-controlled studies evaluating the treatment response to oral administration of low-dose MTX (12.5-22.5 mg/wk) in active CD, MTX did not demonstrate a superior efficacy over placebo. ${ }^{75,76}$ However, further investigation for the appropriate dose and route of MTX in active CD is needed, because of the limited number of patients included in these studies.

\section{CD Refractory to Medical Treatment}

13. Surgical treatment should be considered in cases that are refractory to medical therapy. Surgical decision making should be done with full communication with gastroenterologists, surgeons, and the patient (quality of evidence, very low; classification of recommendation, weak).

- Level of agreement: strongly agree 61\%, agree 34\%, uncertain 5\%, disagree 0\%, strongly disagree 0\%

14. In case of primary nonresponse to anti-TNF, reevaluation of symptoms and change of treatment are necessary (quality of evidence, low; classification of recommendation, no specific recommendation).

- Level of agreement: strongly agree 42\%, agree 58\%, uncertain $0 \%$, disagree $0 \%$, strongly disagree $0 \%$

15. Although testing of the serum anti-TNF trough level or antibodies to anti-TNF were reported to be useful for optimizing anti-TNF therapy or identifying cause of primary nonresponse or secondary loss of response, further study is required (quality of evidence, low; classification of recommendation, weak).

- Level of agreement: strongly agree 13\%, agree 83\%, uncertain 4\%, disagree 0\%, strongly disagree 0\%

16. In patients who are intolerant or not responsive to one anti-TNF therapy, a different anti-TNF agent may be used (quality of evidence, infliximab [high], adalimumab [low]; classification of recommendation, infliximab [strong], adalimumab [weak]).

- Level of agreement: strongly agree $7 \%$, agree $80 \%$, uncertain 13\%, disagree $0 \%$, strongly disagree $0 \%$

The primary treatment for $\mathrm{CD}$ is medical therapy, and surgical treatment is usually performed to improve symptoms of complications. ${ }^{35}$ Surgery should be decided after thorough communication among the gastroenterologists, patient, and surgeons.

Biologics including anti-TNF agents are the most potent among the currently available drugs and very effective in inducing remission in patients with CD who are not responsive to corticosteroids or immunomodulators. Therefore, failure of biologic treatments may mean failure of medical treatment. There is no consensus about the definition of primary nonresponse, although it is generally accepted as the absence of a response to anti-TNF induction therapy. Concerning the time point for defining nonresponse, it is recommended that primary nonresponse should not be assessed before 8 to 12 weeks following the initial dosing of anti-TNF agents. ${ }^{6}$ The incidence of primary nonresponse was reported to be $20 \%$ to $40 \%$ in clinical studies and $10 \%$ to $20 \%$ in observational studies. ${ }^{77}$ Several factors are known to predict primary nonresponse, including a disease duration 
of $>2$ years, small-bowel involvement, smoking, and normal CRP. Several genetic polymorphism (IBD5, etc.) were also reported as associated factors. ${ }^{6,69,78-81}$ Therefore, smoking cessation is mandatory before treatment to prevent primary nonresponse to anti-TNF therapy. Moreover, it is necessary to evaluate whether there is indeed any evidence of active inflammation, such as endoscopic ulcers or elevated CRP, and the association between inflammatory activity and patient symptoms. Nonresponse to anti-TNF therapy is more likely to occur when symptoms are associated with other noninflammatory causes, such as a fibrotic stricture. ${ }^{82,83}$ Change to other anti-TNF drug may be an appropriate choice in case of primary nonresponse, as $50 \%$ to $60 \%$ of those patients respond to other anti-TNF drugs.

Although the mechanism of primary nonresponse has not yet been clearly been identified, pharmacokinetic factors may play an important role, which means that the lack of response is caused by the accelerated clearance of the drug from systemic circulation and/or local tissue. ${ }^{84,85}$ Immunogenicity, the formation of anti-drug antibody (ADA), has been in the spotlight because it has been reported that binding of ADA to the drug results in increased clearance of the drug, leading to unfavorable clinical outcomes. In fact, in many studies, the response to treatment was poor with lower serum concentrations of anti-TNF drug and in the presence of ADA. In comparison with patients who received $80 / 40 \mathrm{mg}$, those who received $160 / 80 \mathrm{mg}$ for the induction of remission showed higher adalimumab serum concentration at week 4 (11.6 vs. $3.6 \mu \mathrm{g} / \mathrm{mL} ; P=0.0001)$ and lower incidence of primary nonresponse (OR, 0.02; 95\% CI, 0.003-0.2; $P=0.012) .{ }^{86}$ However, further study is required on the usefulness of ADA detection and anti-TNF drug serum level in the treatment, prevention, or prediction of primary nonresponse ${ }^{85,87}$ Other suggested mechanisms of primary nonresponse include non-TNF inflammatory pathway, rapid consumption of anti-TNF drug owing to severe inflammation, and presence of inherent antibodies to the anti-TNF drug before exposure to the drug. ${ }^{84,85}$

Vedolizumab, a monoclonal antibody to $\alpha_{4} \beta_{7}$ integrin, has a totally different mechanism to the previous anti-TNF drugs, and has an anti-inflammatory effect by blocking lymphocyte migration to the gastrointestinal tract. In the GEMINI 3 study on vedolizumab induction therapy for patients with moderate to severe CD who failed treatment with previous anti-TNFs, vedolizumab induction therapy did not show a difference at 6 weeks in comparison with placebo (15.2\% vs. $12.1 \% ; P=0.433)$, but the remission rate at 10 weeks was significantly higher than with placebo ( $26.6 \%$ vs. $12.1 \%$; $P=0.001$; RR, 2.2; 95\% CI, 1.3-3.6)..$^{88}$ Another biologic agent, ustekinumab, is a monoclonal antibody to the p40 subunit of interleukin-12 and interleukin-23, which was approved for the treatment of psoriasis but has recently been shown to be efficacious in intractable CD. ${ }^{89}$ In the UNITI- 1 study, in which 741 patients with moderate CD who had a history of anti-TNF use (primary nonresponse, secondary loss of response, or intolerance to anti-TNF) were randomized to ustekinumab $130 \mathrm{mg}, 6 \mathrm{mg} / \mathrm{kg}$ or placebo, the response rates of ustekinumab $130 \mathrm{mg}$ and $6 \mathrm{mg} / \mathrm{kg}$ group were $34.3 \%$ and $33.7 \%$ at 6 weeks respectively, which was higher than that of the placebo group (21.5\%) (according to the dosage group, $P=0.002$ and $P=0.003$, respectively) ${ }^{89}$ These results suggest that vedolizumab and ustekinumab may be used as a second-line therapy in patients with primary nonresponse or intolerance to anti-TNF therapy.

For cases that are intolerant to a specific anti-TNF drug, other anti-TNF drugs may show effectiveness. The Gauging Adalimumab Efficacy in Infliximab Nonresponders (GAIN) study including patients with CD who were intolerant or nonresponsive to infliximab demonstrated significantly higher remission and response rates (21\% vs. $7 \%, 52 \%$ vs. $34 \% ; P<0.05)$ at week 4 in patients who received adalimumab 160/80 mg for induction of remission in comparison with the placebo group, and a similar effect was observed across the infliximab-intolerant and infliximab-nonresponse groups. $^{90}$

\section{MAINTENANCE THERAPY OF CD}

\section{Maintenance Therapy after 5-ASA-induced Remission}

17. 5-ASA has fewer adverse events, but has limited effect in maintaining the remission of $C D$ (quality of evidence, high; classification of recommendation, strong).

- Level of agreement: strongly agree 33\%, agree 65\%, uncertain $2 \%$, disagree $0 \%$, strongly disagree $0 \%$

There are few studies on maintenance therapy after remission induced by 5-ASA. In a meta-analysis comparing the efficacy of 5-ASA and placebo for maintenance therapy in patients with remission induced by medical treatment, there was no significant difference between the two groups. ${ }^{91}$ Although seven studies included in this meta-analysis were not specific to patients who achieved remission with 5-ASA alone, all subjects achieved clinical remission from medical treatment and were maintained for at least a month without corticosteroids or immunomodulators before enrollment. On the other hand, in a recent meta-analysis, treatment with 
$\mathrm{pH}$ 7-dependent mesalamine significantly reduced the risk of relapse in patients with either medically or surgically induced remission in comparison with the placebo group (OR, 0.28; 95\% CI, 0.12-0.65; $P=0.003),{ }^{92}$ whereas $\mathrm{pH}$ 6-dependent mesalamine or controlled-release mesalamine did not demonstrate significant differences. ${ }^{92}$ Although further studies are required, 5-ASA maintenance therapy may be considered in case of mild CD with 5-ASA-induced remission.

\section{Maintenance Therapy after Corticosteroid-induced Remission}

18. Systemic corticosteroids and budesonide are not recommended as maintenance therapy for CD (quality of evidence, high; classification of recommendation, strong).

- Level of agreement: strongly agree 75\%, agree 23\%, uncertain $0 \%$, disagree $2 \%$, strongly disagree $0 \%$

19. Thiopurine is recommended for maintenance therapy in the case of corticosteroid-induced remission (quality of evidence, moderate; classification of recommendation, strong).

- Level of agreement: strongly agree 48\%, agree 50\%, uncertain 2\%, disagree 0\%, strongly disagree 0\%

Systemic corticosteroids are effective in inducing but ineffective in maintaining the remission of CD. In three randomized double-blind placebo-controlled studies, the ORs (95\% $\mathrm{CI}$ ) for relapse in the corticosteroid groups to the placebo groups were $0.71(0.39-1.31), 0.82(0.47-1.43)$, and 0.72 (0.38-1.35) for 6, 12, and 24 months, respectively. Moreover, long-term use of corticosteroids caused many adverse effects, including osteoporosis and cataracts. ${ }^{61}$ Budesonide showed no differences in preventing relapse in comparison with placebo (RR, 0.93; 95\% CI, 0.83-1.04). ${ }^{93}$

Thiopurine is effective for maintenance therapy if remission is induced by corticosteroid therapy ${ }^{94,95}$ Candy et al. ${ }^{94}$ demonstrated a significantly lower relapse rate at 1 year in the AZA $2.5 \mathrm{mg} / \mathrm{kg} /$ day group than in the placebo group in patients with cortico steroid-induced remission (RR, 0.47; 95\% CI, 0.28-0.77). In contrast, the National Cooperative Crohn's Disease Study (NCCDS) showed no difference in relapse rate between placebo and AZA $1 \mathrm{mg} / \mathrm{kg} /$ day maintenance therapy for 2 years. However, the result needs to be interpreted carefully as the AZA doses were relatively low and many patients with surgically induced remission rather than corticosteroid-induced remission were included in the NCCDS ${ }^{60}$ Recent guidelines for treatment of CD, therefore, recommend thiopurine maintenance therapy for patients with corticosteroid-induced remission. ${ }^{10}$

\section{Maintenance Therapy after Anti-tumor Necrosis Factor-Induced Remission}

20. Anti-TNF agents are recommended as maintenance therapy for cases with anti-TNF-induced remission (quality of evidence, high; classification of recommendation, strong).

- Level of agreement: strongly agree 80\%, agree 20\%, uncertain $0 \%$, disagree $0 \%$, strongly disagree $0 \%$

21. Scheduled anti-TNF maintenance therapy is recommended rather than episodic therapy (quality of evidence, high; classification of recommendation, strong).

- Level of agreement: strongly agree 87\%, agree 13\%, uncertain $0 \%$, disagree $0 \%$, strongly disagree $0 \%$

22. After inducing remission by using combined anti-TNF and thiopurine therapy, an anti-TNF monotherapy or combination therapy of the two drugs may be considered depending on the clinical features of the patient and the adverse effects of the drugs (quality of evidence, low; classification of recommendation, weak).

- Level of agreement: strongly agree 30\%, agree 70\%, uncertain $0 \%$, disagree $0 \%$, strongly disagree $0 \%$

The efficacy of anti-TNF maintenance therapy was superior to that of placebo in patients with remission induced by anti-TNF agents. ${ }^{96}$ In a meta-analysis on the efficacy of infliximab maintenance therapy in patients who responded to infliximab, infliximab was superior to placebo in maintaining clinical remission (RR, 2.50; 95\% CI, 1.64-3.80), ${ }^{40,41,96}$ maintaining clinical response (RR, 2.19; 95\% CI, 1.27-3.75), ${ }^{40,41,96}$ and the corticosteroid-sparing effect (RR, 3.13; 95\% CI, $1.25-7.81){ }^{41,96}$ Meanwhile, there was no difference in remission rate between infliximab $5 \mathrm{mg} / \mathrm{kg}$ and $10 \mathrm{mg} / \mathrm{kg}^{41}$ Adalimumab was also superior to placebo in maintaining clinical remission (RR, 3.28; 95\% CI, 2.13-5.06), maintaining clinical response (RR, 2.69; 95\% CI, 1.88-3.86), and the corticosteroid-sparing effect (RR, 4.25; 95\% CI, 1.57-11.47) ${ }^{79,96} \mathrm{In}$ addition, the admission rate and surgery rate during the first year of maintenance therapy were lower in the adalimumab group than in the placebo group. ${ }^{97}$ However, there was no difference in remission rates between the adalimumab 40 mg every other week (EOW) and every week (EW) groups. ${ }^{79}$ Certolizumab maintenance therapy was also superior to placebo in maintaining clinical remission (RR, 1.68; 95\% CI, $1.30-2.16)$ and response (RR, 1.74; 95\% CI, 1.41-2.13). ${ }^{96,98}$

In the post hoc analysis of A Crohn's Disease Clinical Trial Evaluating Infliximab in a New Long-term Treatment Regimen (ACCENT) I study, scheduled administration of infliximab was superior to episodic infliximab therapy in terms of CDAI decrease, clinical response rate, and clinical remission rate. ${ }^{99}$ Moreover, in the scheduled treatment group, 
the anti-infliximab antibody formation rate, ${ }^{99,100}$ admission rate, and surgery rate related to $\mathrm{CD}$ were lower than in the episodic therapy group. ${ }^{99}$ In addition, the rate of complete mucosal healing was higher in the scheduled infliximab therapy group than in the episodic therapy group scheduled after response to infliximab induction therapy (54\% vs. 7\%; $P=0.007$ ), which proved the superiority of scheduled administration of infliximab. ${ }^{101}$ Overall, episodic administration of anti-TNF drugs increases the risk of relapse, especially in patients who had failed treatment with immunomodulators. ${ }^{13}$

According to the SONIC study, the infliximab and AZA combination therapy was superior to both infliximab monotherapy and AZA monotherapy in terms of clinical remission and corticosteroid-free remission, which persisted until 50 weeks. However, the efficacy of maintenance therapy was not the primary outcome variable of the SONIC study. ${ }^{69}$ In a prospective RCT with patients who were treated with a combination of infliximab and immunomodulators (thiopurines or MTX), and were maintained in clinical remission for $\geq 6$ months, there was no difference in maintaining the infliximab efficacy until 104 weeks between the immunomodulator maintenance group and the immunomodulator withdrawal group. ${ }^{102}$ However, it was reported that the CRP level was higher and the trough level of infliximab was lower at 104 weeks in the withdrawal group than in the maintenance group, suggesting the usefulness of maintaining immunomodulators. ${ }^{102}$ There is a report that even in scheduled infliximab maintenance therapy for IBD, scheduled concomitant administration of immunomodulators was superior to infliximab alone in terms of relapse, perianal complications, and switch to adalimumab because of infliximab failure. ${ }^{103} \mathrm{In}$ multivariate analysis, concomitant administration of immunomodulators reduced the risk of IBD relapse (OR, 0.52; 95\% CI, 0.35-0.79).$^{103}$ In a recent small-scale study, withdrawal of AZA within 6 months of combination therapy was associated with a loss of response to anti-TNF agents. ${ }^{104}$ On the other hand, in pediatric patients with CD with remission induced by a combination of infliximab and immunomodulators, there were no differences in the rate of loss of response, pediatric CDAI, and simple endoscopic score for CD between the withdrawal group $(\mathrm{n}=39$, patients who discontinued immunomodulators at week 26) and the maintenance group $(\mathrm{n}=45)$ until week $54 .{ }^{105}$ A meta-analysis of six randomized placebo-controlled studies showed that the combination of immunomodulators with anti-TNF agents and treatment with anti-TNF agent alone in patients who were previously treated with immunomodulators demonstrated no differences in terms of clinical response and response at 6 months: infliximab (combination therapy vs. monotherapy: remission rate, $\mathrm{OR}=1.73,95 \% \mathrm{CI}=0.97-3.07$; response rate, $\mathrm{OR}=4.14$, 95\% CI=0.52-32.68), adalimumab (combination therapy vs. monotherapy: remission rate, $\mathrm{OR}=0.88,95 \% \mathrm{CI}=0.58-1.35$; response rate, $\mathrm{OR}=0.84,95 \% \mathrm{CI}=0.55-1.27$ ), certolizumab (combination therapy vs. monotherapy: remission rate, $\mathrm{OR}=0.93,95 \% \mathrm{CI}=0.65-1.34$; response rate, $\mathrm{OR}=0.97,95 \%$ $\mathrm{CI}=0.66-1.43) .{ }^{106}$ In a meta-analysis of adalimumab studies, the adalimumab monotherapy group and the immunomodulator combination therapy group did not differ in the clinical remission rate (monotherapy vs. combination therapy: OR, 1.08; 95\% CI, 0.79-1.48) and response rate (monotherapy vs. combination therapy: OR, 1.21; 95\% CI, 0.74-1.99) at 12 months. ${ }^{70}$ In addition, there was also no difference in the need for increasing the dose of adalimumab because of loss of response (mono month vs. combination therapy: OR, 1.13; 95\% CI, 0.69-1.89)..$^{70}$ Although the incidence of infusion reactions was lower in the immunomodulator combination therapy group than in the infliximab monotherapy group (OR, 0.46; 95\% CI, 0.26-0.79), ${ }^{106}$ there were no significant differences in the incidences of severe infection, malignancy, and mortality. ${ }^{106}$ Collectively, studies comparing the efficacy of the anti-TNF monotherapy with immunomodulator combination therapy for the maintenance of remission in CD showed variable results and do not provide enough evidences to recommend any one strategy.

\section{Maintenance Therapy after Methotrexate-Induced Remission}

23. If remission is induced by intramuscular MTX, MTX can be used as a maintenance therapy (quality of evidence, high; classification of recommendation, strong).

- Level of agreement: strongly agree 19\%, agree 77\%, uncertain $4 \%$, disagree $0 \%$, strongly disagree $0 \%$

24. If a patient does not tolerate or does not respond to thiopurines, or if thiopurines are contraindicated, MTX can be considered as a maintenance agent (quality of evidence, moderate; classification of recommendation, strong).

- Level of agreement: strongly agree 18\%, agree 78\%, uncertain $4 \%$, disagree $0 \%$, strongly disagree $0 \%$

When patients with $\mathrm{CD}$ with remission that was induced by weekly intramuscular injection of MTX $25 \mathrm{mg}$ were randomly assigned to the weekly intramuscular MTX $15 \mathrm{mg}$ injection group or to the placebo group, $65 \%$ of the MTX group and $39 \%$ of the placebo group showed clinical remission at 40 weeks ( $P=0.04$ ) (RR 1.67, 95\% CI 1.05-2.67). In addition, fewer patients in the MTX group (28\%) required prednisolone for relapse than those in the placebo group (28\% vs. 
$58 \%, P=0.01) .{ }^{107,108}$ Although there is no direct comparison with intramuscular administration, subcutaneous MTX was also reported to be efficacious and safe in treating IBD. ${ }^{109}$ Although there is no direct comparison of MTX with thiopurine in maintaining the remission of CD, MTX is considered a second-line immunomodulator in cases of intolerance or contraindication to thiopurines or when patients are unresponsive to thiopurines., ${ }^{8,110}$

\section{Relapse during Maintenance Therapy}

25 . In case of relapse during maintenance therapy with immunomodulators, anti-TNF agents are recommended (quality of evidence, high; classification of recommendation, strong).

- Level of agreement: strongly agree 72\%, agree 28\%, uncertain $0 \%$, disagree $0 \%$, strongly disagree $0 \%$

26. If the therapeutic efficacy of infliximab $(5 \mathrm{mg} / \mathrm{kg})$ is decreased or insufficient, shortening the interval of infusion or increasing the dose up to $10 \mathrm{mg} / \mathrm{kg}$ can be considered (quality of evidence, high; classification of recommendation, strong).

- Level of agreement: strongly agree 72\%, agree 28\%, uncertain 0\%, disagree 0\%, strongly disagree 0\%

27. If the therapeutic efficacy of adalimumab $(40 \mathrm{mg}$ biweekly) is decreased or insufficient, weekly adalimumab administration can be considered (quality of evidence, high; classification of recommendation, strong).

- Level of agreement: strongly agree 70\%, agree 30\%, uncertain $0 \%$, disagree $0 \%$, strongly disagree $0 \%$

28. If the therapeutic efficacy is insufficient after shortening the interval of administration or increasing the dose of anti-TNF agents, switching to another anti-TNF agent can be considered (quality of evidence, high; classification of recommendation, strong).

- Level of agreement: strongly agree 36\%, agree 64\%, uncertain $0 \%$, disagree $0 \%$, strongly disagree $0 \%$

In the case of relapse during maintenance therapy, causes other than insufficient control of inflammation, such as complications including fibrous stenosis, abscess, fistula, dietary intolerance, or combined IBS, should be ruled out. ${ }^{13}$ In addition, comprehensive evaluation including imaging, endoscopic evaluation, blood tests such as CRP, and fecal calprotectin is needed. ${ }^{13}$

Several studies have shown that secondary nonresponse to anti-TNF agents developed in $30 \%$ to $40 \%$ of patients after the first year of treatment. ${ }^{41,79,98}$ In the ACCENT I study, 88\% of patients who lost response during maintenance therapy following primary response to infliximab reestablished response after increasing dosage with $10 \mathrm{mg} / \mathrm{kg} .{ }^{99}$ Other studies reported that $75.9 \%$ of patients regained response after an increase in dose, decrease in infusion interval, or the combination of both, when they showed a secondary nonresponse to infliximab. ${ }^{111}$ High trough concentrations of infliximab have been associated with a more durable response to infliximab; however, low trough concentrations have been associated with a potential loss of response. ${ }^{6}$ In addition, higher rates of clinical remission ( $82 \%$ vs. $6 \% ; P<0.001)$ and higher rates of endoscopic improvement ( $88 \%$ vs. $33 \% ; P<0.001$ ) were associated with a detectable trough concentration of serum infliximab in patients with $\mathrm{CD}$ receiving infliximab maintenance therapy. ${ }^{100} \mathrm{~A}$ post hoc analysis of the Crohn's Trial of the Fully Human Antibody Adalimumab for Remission Maintenance (CHARM) study showed that clinical response with a CDAI decrease $\geq 70$ points could be achieved after open-label adalimumab $40 \mathrm{mg}$ EW therapy in $63.4 \%$ (45 of 71) of those with a lack of response or flares during adalimumab $40 \mathrm{mg}$ EOW therapy. ${ }^{112}$ In a Belgian study, the response rate after switching to adalimumab $40 \mathrm{mg}$ EW therapy was $71.6 \%$ (73 of 102).$^{86}$ Discontinuation of adalimumab was significantly related to low trough serum concentration of adalimumab during maintenance therapy. ${ }^{86}$

No response to increasing dose or shortening the interval of anti-TNFs can be managed by switching to a different antiTNF agent. However, there is a lower chance of a therapeutic response to a second anti-TNF agent compared with the first anti-TNF. ${ }^{6}$ Switching to an agent with a different mechanism of action is logical in case of failure to all available anti-TNF agents. ${ }^{6}$ In the GEMINI 2 trial, which evaluated the efficacy of monoclonal antibody to the $\alpha_{4} \beta_{7}$ integrin vedolizumab in patients with $\mathrm{CD}$, responders to induction therapy were randomized into vedolizumab every 8 weeks, vedolizumab every 4 weeks, or placebo groups for up to 52 weeks. ${ }^{113}$ As a result, the remission rate in a prior anti-TNF-failure group was $28.0 \%$ in the vedolizumab every 8 weeks group, $27.3 \%$ in the vedolizumab every 4 weeks group, and $12.8 \%$ in the placebo group at 52 weeks. The both vedolizumab-treated groups showed higher probabilities of clinical remission than the placebo group (vedolizumab every 8 weeks group vs. placebo group, $P=0.01$ and vedolizumab every 4 weeks group vs. placebo group, $P=0.02) .{ }^{113}$

Meanwhile, in the UNITI-1 study, which also included patients with secondary loss of response to anti-TNF, ustekinumab, a monoclonal antibody to interleukin-12/23, was superior to placebo for inducing clinical response of CD. ${ }^{89}$ Furthermore, when only patients who responded to ustekinumab among UNITI- 1 and UNITI-2 subjects were randomly assigned to ustekinumab $90 \mathrm{mg}$ administration at 8 weeks or 12 weeks interval, or placebo, the remission rate at week 44 were significantly higher in the ustekinumab 
group (53.1\% in 8 weeks group and $48.8 \%$ in 12 weeks group) than in the placebo group (35.9\%) (according to the interval $P=0.005$ and $P=0.04$, respectively) ${ }^{89}$ These results suggest that vedolizumab and ustekinumab can be effective alternative agents in patients with loss of response to antiTNF.

\section{TREATMENT OF CD BASED ON DISEASE LOCATION}

\section{CD Localized in the Distal Ileum}

29. For CD limited to the distal ileum, early surgery may be considered as an alternative to medical therapy, after assessing the risks and benefits of medical and surgical therapy, risk of postoperative recurrence, and patient preference (quality of evidence, low; classification of recommendation, weak).

- Level of agreement: strongly agree 13\%, agree 76\%, uncertain 9\%, disagree 2\%, strongly disagree 0\%

It is reported that $25 \%$ to $30 \%$ of CD involves the distal ileum alone or with the cecum. ${ }^{114}$ For CD limited to the distal ileum, early surgical resection may be an alternative to medical therapy; however, there is no comparative study of these two treatments in this patient group.

Regarding the postoperative course of patients with CD involved to the ileum, a retrospective review of 56 patients who underwent ileo-colonic resection showed a clinical relapse rate of $52 \%$, a hospitalization rate of $9 \%$, and a reoperation rate of $29 \%$, during 10.5 years follow-up period ${ }^{115}$ In another study, when 55 patients were followed for 6.8 years after ileocolonic resection, the clinical relapse rate was $38 \%$ and reoperation rate was $9 \%$, and the quality of life of CD patients was equal to that of the general population. ${ }^{116}$ As described above, CD localized around the distal ileum could maintain long-term remission after surgery in a substantial number of patients, and the quality of life was also positively influenced by removing the involved bowel segment. In addition, surgical techniques have improved over the past 2 decades and minimally invasive procedures such as laparoscopic surgery have been widely introduced in CD, reducing surgical morbidity and speeding up patient recovery. ${ }^{117}$

The results of aforementioned studies suggest that early surgical treatment may be considered as an alternative to medical treatment for patients with involvement of the distal ileum alone, and the risks and benefits of medical and surgical treatments, as well as the risk of postoperative recurrence should be discussed before treatment methods are selected. In addition, medical treatment may be beneficial in patients with active fistulae or severe extra-intestinal symptoms, whereas surgical intervention may be considered for fibrosing stricture of the distal ileum. ${ }^{117}$ In the future, prospective studies should be conducted to compare and evaluate the quality of life and cost-effectiveness of each treatment method.

\section{CD of the esophagus, stomach, or duodenum}

30. Use of proton pump inhibitors is considered for symptomatic upper gastrointestinal CD (quality of evidence, very low; classification of recommendation, weak).

- Level of agreement: strongly agree 15\%, agree 76\%, uncertain 9\%, disagree 0\%, strongly disagree 0\%

31. Anti-TNF therapy is considered if there is no response to systemic corticosteroid therapy (quality of evidence, very low; classification of recommendation, weak).

- Level of agreement: strongly agree 30\%, agree 65\%, uncertain $5 \%$, disagree $0 \%$, strongly disagree $0 \%$

32. Endoscopic dilatation or surgery is considered for upper gastrointestinal CD with gastrointestinal obstruction (quality of evidence, very low; classification of recommendation, weak).

- Level of agreement: strongly agree 27\%, agree 73\%, uncertain $0 \%$, disagree $0 \%$, strongly disagree $0 \%$

CD isolated to the esophagus, stomach or duodenum is very rare, ${ }^{118}$ and $\mathrm{CD}$ patients with stomach and duodenal lesions generally also have distal small bowel or colonic lesions. The management of upper gastrointestinal CD is determined by the extent of the small intestinal and colonic involvement, except when a stricture is present..$^{35,118,119} \mathrm{~A}$ proton pump inhibitor is usually considered because gastric acid may worsen gastro-duodenal ulcers in the upper gastrointestinal tract. ${ }^{119-122}$ Since the general form of the 5-ASA agent does not act on the upper gastrointestinal mucosa, attempts have been made to prepare and administer the tablet in powdered form, but the efficacy and safety of such administration has not been fully demonstrated. ${ }^{11}$ Endoscopic balloon dilatation may be considered in cases of upper gastrointestinal obstruction, ${ }^{123,124}$ and as in other cases with bowel strictures, endoscopic treatment can be considered if the stricture is single and short. ${ }^{125}$ If the symptoms of stenosis are progressive despite medical and endoscopic treatment, surgical treatments including bypass, resection, or strictureplasty can be performed. ${ }^{126}$ 


\section{TREATMENT OF CD WITH STRICTURE OR FISTULA}

\section{Treatment of Stricturing CD}

33. Administer systemic corticosteroids if the stricture is accompanied with severe inflammation (quality of evidence, very low; classification of recommendation, weak).

- Level of agreement: strongly agree 18\%, agree 71\%, uncertain $9 \%$, disagree $2 \%$, strongly disagree $0 \%$

34. If there is no improvement after drug therapies or decompression, consider endoscopic balloon dilatation if stricture is endoscopically accessible, short, straight, and single (quality of evidence, very low; classification of recommendation, weak).

- Level of agreement: strongly agree 22\%, agree 76\%, uncertain 0\%, disagree 2\%, strongly disagree 0\%

35. If there is no improvement on medical treatment, consider surgery (quality of evidence, very low; classification of recommendation, weak).

- Level of agreement: strongly agree 40\%, agree 58\%, uncertain $2 \%$, disagree $0 \%$, strongly disagree $0 \%$

Gastrointestinal stricture in CD may be caused by mucosal edema due to acute inflammation or fibrosis of the entire layer of the gastrointestinal wall due to chronic inflammation. According to expert opinion, inflammation-induced strictures can be improved on medical treatment, such as with the use of corticosteroids. ${ }^{127}$ In addition, the possibility of stenosis due to fibrosis should be considered if there is no improvement on anti-inflammatory treatment, and the feasibility of endoscopic balloon dilatation should be considered after an assessment of the stricture length, number of stricture sites, and ulceration status. Endoscopic balloon dilatation is preferably performed after the inflammation and ulceration have improved. In a retrospective study of 59 patients with symptomatic stricturing CD (53 anastomotic strictures and 6 nonanastomotic strictures), $41 \%$ of patients showed symptomatic improvement after endoscopic balloon dilatation over a follow-up period of 29 months ( $17 \%$ of patients underwent only one dilatation). ${ }^{128}$ In a prospective study of 55 patients with CD with symptomatic strictures, $68 \%$ of the patients were able to avoid surgery for 5 years after balloon dilatation. ${ }^{124}$

Anti-TNF agents are not recommended for CD with fibrosing strictures. If objective evidences of active inflammation, which includes elevated CRP and endoscopic or radiologic active lesions, is not present, patients generally show poor response to anti-TNF agents. Meanwhile, anti-TNF is not contraindicated in all strictures, and inflammatory strictures may be improved using anti-TNF agents. ${ }^{122}$ Nevertheless, attention should be paid when using anti-TNF agents in cases of CD with stricturing lesion, since rapid inflammatory healing with this drug can worsen the stricture. In an observational study ${ }^{129}$ that analyzed the TREAT registry data, infliximab-treated patients had more strictures than patients who were treated with other drugs( 1.95 vs. 0.99 per 100 patient-years, $P<0.001$ ), but the severity of infliximabtreated patients was higher and infliximab was not identified as an independent risk factor for stricture in multivariate analysis. In another observational study of 36 patients with CD who received anti-TNF agents, partial or complete gastrointestinal obstructions occurred in $8 \%$ of patients during an average follow-up period of 23 months, and all these obstructions occurred among patients with underlying stricturing behavior. ${ }^{130}$ Since this observational study lacked a control group, it is difficult to determine whether the cause of obstruction was related to anti-TNF or the patients' clinical characteristics; however, caution is needed when using antiTNF in patients with $\mathrm{CD}$ and stricture.

\section{Treatment of Fistulizing CD}

Fistulizing $\mathrm{CD}$ is difficult to treat and shows poor prognosis. Fistulizing CD includes entero-enteric, enterocutaneous, perianal fistula, and fistulas between the intestine and other organs. It is difficult to establish optimal treatment guideline for fistulizing CD because of diagnosis and treatment complexity and the fact that high-level clinical data for fistulizing $\mathrm{CD}$ are lacking. Important points to consider when planning treatment for fistulas associated with CD include fistula origin and anatomical location, evaluation of concomitant luminal inflammation and stricture, identification of local sepsis (abscess), involved organ and associated systemic symptoms, impact on quality of life, and nutritional status.

A clinical or radiological evaluation can be used to assess treatment response of fistula. In daily practice, evaluation methods based on clinical indicators such as decreased fistula drainage volume and symptom improvement are mainly used. The fistula drainage assessment (FDA), which was proposed to objectively evaluate the activity and therapeutic response of fistulas, has been used in several clinical trials. ${ }^{131}$ Regarding the FDA method, a fistula is considered active when purulent discharge is drained with gentle finger compression around it but in remission when no discharge is observed. A clinical response is defined as a $\geq 50 \%$ reduction in the number of fistulas with discharge, while fistula closure or clinical remission is defined as no discharge after gentle finger compression in any fistula. ${ }^{131}$ 
Meanwhile, the Perianal Disease Activity Index (PDAI) was proposed for the exclusive assessment of perianal fistulas. The PDAI consists of five indices (activity restriction/ pain, sexual activity restriction, fistula discharge, perianal disease type, and induration degree) that can comprehensively evaluate perianal fistula activity. However, the PDAI is difficult to use clinically because of its complexity and has not yet been validated. ${ }^{132,133}$

\section{1) Treatment of Perianal Fistula}

The cumulative incidence of a perianal fistula in CD is approximately $10 \%$ at 1 year, $15 \%$ at 5 years, and $20 \%$ at 10 years. ${ }^{134}$ The risk of developing perianal fistula is higher in colonic disease at up to $92 \%$ with rectal involvement. ${ }^{134}$ In a multicenter study in Korea, 36\% of patients had a history of perianal fistula, while $28 \%$ had concomitant perianal fistulas at the time of the CD diagnosis. ${ }^{135-137}$

The short term goals of treating perianal fistula are abscess drainage and reduction of symptoms. The long term goals are resolving fistula discharge, improvement in quality of life, fistula healing, resolution of fecal incontinence, and avoiding proctectomy with stoma. ${ }^{12}$ The treatment of perianal fistula varies by fistula location, presence of abscess, and disease activity. Although many clinical studies investigated the treatment of perianal fistulas in $\mathrm{CD}$, there are limitations to developing evidence-based approaches because of the lack of comparative studies on medical or surgical treatment alone or in combination. Multidisciplinary approaches by gastroenterologists, radiologists, and surgeons can be helpful for determining optimal management for perianal fistula, especially in cases in which both medical and surgical treatments are considered.

It is important to accurately classify fistula type to ensure the proper management of perianal fistula, and the classification standard of the American Gastroenterological Association that classifies the fistula as simple or complex, is widely used. ${ }^{133}$ A simple fistula is located below the dentate line and has one external opening without perianal complications, whereas a complex fistula is located higher than the dentate line, can have multiple fistula openings, and is associated with complications such as perianal abscess, rectal stenosis, rectal inflammation, or rectovesicular or rectovaginal fistula. To ensure accurate fistula diagnosis and classification, pelvic magnetic resonance imaging (MRI), endo-anal ultrasonography, or examination under anesthesia are used, while endoscopy is also needed to evaluate rectal inflammation presence and severity.

\section{(1) Simple perianal fistula}

36. Simple asymptomatic perianal fistulas do not require treatment (quality of evidence, very low; classification of recommendation, no specific recommendation).

- Level of agreement: strongly agree $18 \%$, agree $62 \%$, uncertain 13\%, disagree 7\%, strongly disagree $0 \%$

37. For symptomatic simple perianal fistulas, metronidazole $(750-1,500 \mathrm{mg} /$ day $)$ or ciprofloxacin $(1,000 \mathrm{mg} /$ day $)$ is recommended (quality of evidence, low or very low; classification of recommendation, strong).

- Level of agreement: strongly agree $29 \%$, agree $71 \%$, uncertain $0 \%$, disagree $0 \%$, strongly disagree $0 \%$

38. For symptomatic simple perianal fistulas, noncutting seton or fistulotomy is recommended (quality of evidence, low or very low; classification of recommendation, strong).

- Level of agreement: strongly agree $27 \%$, agree $71 \%$, uncertain $0 \%$, disagree $2 \%$, strongly disagree $0 \%$

Medical or surgical treatment should be considered for symptomatic simple perianal fistula, and it is advisable to establish a treatment plan in consultation with a colorectal surgeon. Surgical treatment is generally followed by the initial medical treatment according to treatment response, but concomitant treatments can be considered depending on the clinician's experience and preference.

Antibiotics, most commonly metronidazole and ciprofloxacin, are considered the first-line treatment for simple perianal fistulas in CD. Although antibiotics improve fistula symptoms and may contribute to fistula healing, they do not induce complete fistula closure and the fistula often deteriorates after their discontinuation. In addition, metronidazole should be used cautiously because of adverse events associated with their long-term use. The literature on the use of antibiotics is limited and it consists mostly of small case series. Studies using metronidazole have shown that $\geq 50 \%$ of patients responded to treatment, with a significant number of fistulas recurring upon discontinuation. ${ }^{138-140}$ In a study comparing the efficacy of ciprofloxacin and metronidazole in 25 patients with CD and fistula, the treatment response at week 10 was $40 \%$ in the ciprofloxacin group, $13.3 \%$ in the metronidazole group, and $12.5 \%$ in the placebo group, and although there was no significant difference among the groups, the numerical value was higher in the ciprofloxacin group $(P=0.430) .{ }^{141}$

Fistulotomy alone is reported to induce remission in $85 \%$ of patients with simple perianal fistula without rectal inflammation. ${ }^{142}$ In contrast, if active inflammation is present in the rectum, the risk of delayed healing or fecal incontinence 
increases after fistulotomy. Therefore, a noncutting seton placement is preferred along with appropriate medical treatment for active inflammation. A seton improves symptoms by maintaining drainage until the inflammation of the fistula tract is resolved by drug therapy and is usually removed when it is judged that the fistula tract has been completely healed. ${ }^{143}$

Although thiopurine may have efficacy against perianal fistulas in CD, no RCT has directly assessed the efficacy of thiopurine against perianal fistula. The basis for the use of thiopurine in perianal fistula in CD is a meta-analysis involving five placebo-controlled studies published in 1995, showing that thiopurine has a significant effect on fistula healing compared to placebo (OR, 4.44; 95\% CI, 1.5-13.2). ${ }^{67}$ However, a recent meta-analysis of three well-designed randomized placebo-controlled studies showed no significant efficacy of AZA and 6-MP on symptom improvement or perianal fistula closure compared with placebo (RR, 2; 95\% CI, 0.67-5.93). ${ }^{68}$ Nevertheless, the number of patients included in the metaanalysis was very small (18 patients) and the healing of the fistula was a secondary endpoint, so further studies are needed. Despite this limited evidence regarding the effect of thiopurine on perianal fistula in $\mathrm{CD}$, thiopurine is believed to have a moderate effect on perianal fistula. ${ }^{12}$ The efficacy of AZA in combination with antibiotic therapy was prospectively investigated with 52 patients with perianal fistula, 83\% of whom had simple fistula. After 8 weeks of antibiotic therapy, patients who received AZA $(n=29)$ were more likely to achieve a response than observation without drug therapy $(\mathrm{n}=19)$ at week 20 ( $48 \%$ vs. $15 \% ; P=0.030$ ). This finding suggests the usefulness of thiopurine for maintaining perianal fistula. ${ }^{144}$

Other immunomodulators have been reported to treat fistulas in patients with CD. Intravenous cyclosporine has been shown to be effective in the treatment of fistulizing $\mathrm{CD}$ with perianal fistulas and the effect was maintained after the switch to oral agents. ${ }^{145,146}$ However, most fistula recurred after discontinuation, and drug toxicity is a concern for longterm use. In a RCT of 46 patients with fistula (including 42 with perianal fistula) that tested tacrolimus efficacy, the treatment response of fistula was higher in the tacrolimus group than in the placebo group ( $43 \%$ vs. $8 \% ; P=0.004$ ), whereas the complete remission rates did not differ between the two groups (tacrolimus group, $10 \%$ vs. placebo group, 8\%; $P=0.860) .{ }^{147}$ For MTX, only small case series has been reported to date. In this report, of the 16 patients with fistula (including nine with perianal fistula), $56 \%$ of patients showed a fistula response after MTX treatment. ${ }^{148}$ In future, a placebo-controlled study is needed to confirm the efficacy of MTX for fistulizing CD.

(2) Complex perianal fistula

39. For complex perianal fistula, the seton procedure is considered (quality of evidence, very low; classification of recommendation, weak).

- Level of agreement: strongly agree 23\%, agree 77\%, uncertain 0\%, disagree 0\%, strongly disagree 0\%

40. For complex perianal fistula, an anti-TNF agent is recommended as a first-line agent in combination with surgery (quality of evidence, high for infliximab and moderate for adalimumab; classification of recommendation, strong).

- Level of agreement: strongly agree 36\%, agree 46\%, uncertain 16\%, disagree $2 \%$, strongly disagree $0 \%$

41. Maintenance therapy of complex perianal fistulas is recommended more than 1 year with an adequate combination of anti-TNF agents, thiopurine, and/or seton (quality of evidence, high; classification of recommendation, strong).

- Level of agreement: strongly agree 29\%, agree 62\%, uncertain $9 \%$, disagree $0 \%$, strongly disagree $0 \%$

The presence of an accompanying perianal abscess should be evaluated at the time of diagnosis of the complex perianal fistula, while surgical drainage should be performed preferentially if a perianal abscess is present. Anti-TNF agent use is recommended in conjunction with adequate drainage using a procedure such as seton.

The first placebo-controlled study including $94 \mathrm{CD}$ patients with fistula (including 85 with perianal fistula) evaluated the efficacy of an infliximab induction regimen for the treatment of fistulas in CD patients. Of them, $68 \%$ who received $5 \mathrm{mg} / \mathrm{kg}$ and $56 \%$ who received $10 \mathrm{mg} / \mathrm{kg}$ achieved the primary end point (a reduction of $\geq 50 \%$ from baseline in the number of draining fistula) compared with $26 \%$ of those in the placebo group $(P=0.002$ and $P=0.020$, respectively). ${ }^{131}$ There was a complete response in $55 \%$ and $38 \%$ of the $5 \mathrm{mg} / \mathrm{kg}$ and $10 \mathrm{mg} / \mathrm{kg}$ groups, respectively, compared with $13 \%$ of the placebo group $(P=0.001$ and $P=0.040$, respectively) ${ }^{131}$ This study confirmed the efficacy of infliximab in the treatment of fistulas in $\mathrm{CD}$, and $5 \mathrm{mg} / \mathrm{kg}$ was found to be an appropriate dosage. The ACCENT II study evaluated the efficacy of infliximab maintenance treatment in patients with fistulizing CD. Patients who responded to the infliximab induction regimen were randomly assigned to receive placebo or infliximab $5 \mathrm{mg} / \mathrm{kg}$ every 8 weeks, and those in the infliximab group showed a higher remission rate than those in the placebo group at 54 weeks ( $36 \%$ vs. $19 \% ; P=0.009)$. ${ }^{149}$ 
No placebo-controlled randomized studies have evaluated the efficacy of adalimumab as a primary outcome variable in patients with fistulizing CD. In the CHARM study, patients who received adalimumab $40 \mathrm{mg}$ EW or EOW showed a significantly higher fistula closure rate compared with those in the placebo group ( $30 \%$ vs. $13 \%$ at week $26, P=0.043 ; 33 \%$ vs. $13 \%$ at week $56, P=0.016) .{ }^{79}$ Moreover, an open-label extension of the CHARM study showed sustained closure in $90 \%$ of 31 patients who continued adalimumab after an additional 1-year follow-up. ${ }^{150}$ In contrast, in two placebocontrolled trials, CLASSIC- 1 and GAIN, adalimumab did not show significant differences in the rates of fistula improvement and remission compared with placebo. However, these studies have the limitation of enrolling fewer patients than the CHARM study. ${ }^{63,90}$

In studies evaluating the efficacy of second-line anti-TNF agents in patients with intractable fistulas that were unresponsive to primary anti-TNF agents, $39 \%$ to $50 \%$ of patients achieved remission of perianal fistula when adalimumab was administered as a second-line agent after a failure of first-line treatment using infliximab. ${ }^{151,152}$ Although there is no report of the efficacy of second-line infliximab after firstline adalimumab failure, this finding suggests that secondline anti-TNF agents may be efficacious in cases with firstline anti-TNF failure.

The Adalimumab for the Treatment of Perianal Fistulas in Crohn's Disease (ADAFI) study assessed the efficacy of a combination of anti-TNF agents and antibiotics. After the adalimumab induction regimen ( $160 \mathrm{mg}$ at week 0 and 80 $\mathrm{mg}$ at week 2), 73 patients with active perianal fistula were randomly assigned to receive ciprofloxacin $500 \mathrm{mg}$ or placebo twice daily for 12 weeks in addition to maintenance treatment of adalimumab $40 \mathrm{mg}$ EOW. Clinical response $(71 \% \mathrm{vs}$. $47 \% ; P=0.047)$ and remission rate $(65 \%$ vs. $33 \% ; P=0.009)$ at week 12 were significantly higher in the combination group than in the adalimumab alone group. However, the difference in fistula closure rates between the two groups was not maintained at week 24 after stopping ciprofloxacin. ${ }^{153}$ With respect to the treatment of perianal fistula in $\mathrm{CD}$, antibiotics can be considered as combination therapy with surgery, immunomodulators, or biologics for the management of CD-related perianal fistula and are used as a bridging agent between remission induction and maintenance therapy.

According to a systematic review of eight comparative studies on the efficacy of combined medical and surgical management for CD-related perianal fistula compared with anti-TNF-based medical therapy or surgical therapy, including various surgical techniques, such as seton, the remission rate of perianal fistula was higher in the combination therapy group than in the single therapy group ( $52 \%$ vs. $43 \%$, respectively). ${ }^{154}$ Although the level of evidence in the included studies is low, this result suggests the usefulness of the combined medical and surgical treatment.

Diverting stoma or proctectomy may be considered for severely complicated perianal fistula, refractory to medical treatment, including anti-TNF agents and seton placement. Based on retrospective data, the early remission rate of diverting stoma is quite high, reaching $81 \%$ in patients with severe refractory fistula, but sustained remission rates decreases gradually to $26 \%-50 \%$ and many patients with a stoma reportedly require proctectomy. ${ }^{155-158}$

Meanwhile, in the first placebo-controlled trial for the efficacy of allogenic fat-derived stem cell injections, in 212 patients with $\mathrm{CD}$, and intractable complex perianal fistulas that were unresponsive to antibiotics, immunomodulators, or anti-TNF, the remission rate at 24 weeks was significantly higher in the stem cell injection group than in the placebo group ( $50 \%$ vs. $34 \%, P=0.024){ }^{159}$ In addition, in 43 patients with complex perianal fistula, including a substantial number of intractable fistulas, when autologous adipose tissuederived stem cell injection was performed, $82 \%$ of the patients $(27 / 33)$ achieved fistula healing at 8 weeks, and remission was maintained in $88 \%$ of those patients at 1 year. ${ }^{160}$ These findings suggest that adipose tissue-derived stem cell therapy may be an alternative treatment for patients with intractable perianal fistula, but future long-term follow-up studies are needed.

\section{2) Treatment of Nonperianal Fistula}

(1) Enterocutaneous fistula

42. For enterocutaneous fistula occurring after surgery, conservative management such as nutritional support is provided after its location is determined, while surgery may be performed if necessary after nutrition status is recovered and a certain period of time passes prior to surgery (quality of evidence, very low; classification of recommendation, no specific recommendation).

- Level of agreement: strongly agree 9\%, agree 82\%, uncertain $7 \%$, disagree $2 \%$, strongly disagree $0 \%$

43. For primary enterocutaneous fistula, surgery (resection of the involved intestine) is generally necessary, although medical treatment may be attempted (quality of evidence, very low, classification of recommendation, no specific recommendation).

- Level of agreement: strongly agree 9\%, agree 73\%, uncertain $11 \%$, disagree $7 \%$, strongly disagree $0 \%$

Nonperianal fistulizing CD includes intestinal-urogenital fistula (bladder, vagina), entero-enteric fistula, and fistula in- 
volving the abdominal wall (enterocutaneous fistula). In this area, there is a lack of high quality evidences, thus establishing evidence-based treatment guidelines is limited.

In a population-based study, the cumulative risk of developing enterocutaneous fistula was $12 \%$ and $24 \%$ after 10 and 20 years, respectively. ${ }^{134}$ The management of enterocutaneous fistula is controversial. Although most enterocutaneous fistula patients required surgery before introduction of antiTNF agent, medical treatment is also considered after antiTNF agent is available. Patients with enterocutaneous fistula have been included in RCTs ${ }^{131,149,150}$ assessing either infliximab or adalimumab (29 enterocutaneous fistula patients [6.8\%] out of 425 total patients) in fistulizing $\mathrm{CD}$, however the number of included patients with enterocutaneous fistula is small, and no data are available in this subgroup.

Meanwhile, a retrospective analysis of 48 patients with enterocutaneous fistula treated with anti-TNF therapy showed that fistula remission was achieved in $33 \%$ of patients during 3-year follow-up. ${ }^{161}$ In this study, it was found that fistula with associated stenosis (hazard ratio [HR], 4.67; 95\% CI, 1.0520.82) and fistulas accompanied by multiple tracts (HR, 5.80; 95\% CI, 1.07-31.5) were independent predictors for failure of anti-TNF therapy. ${ }^{161}$

(2) Entero-urogenital fistula

44. Low anal-introital fistula is mostly asymptomatic and does not require surgery (quality of evidence, very low; classification of recommendation, no specific recommendation).

- Level of agreement: strongly agree 10\%, agree 69\%, uncertain $21 \%$, disagree $0 \%$, strongly disagree $0 \%$

45. Symptomatic female entrogenital fistulas generally require surgery, such as diverting ostomy (quality of evidence, very low; classification of recommendation, no specific recommendation).

- Level of agreement: strongly agree 14\%, agree 75\%, uncertain $9 \%$, disagree $2 \%$, strongly disagree $0 \%$

46. For severe symptomatic rectovaginal fistulas that failed to respond to conservative treatment, surgery such as advancement flap or fecal diversion is considered (quality of evidence, very low; classification of recommendation, no specific recommendation).

- Level of agreement: strongly agree 13\%, agree $82 \%$, uncertain $0 \%$, disagree $5 \%$, strongly disagree $0 \%$

47. Fistulas between the small bowel or sigmoid colon and the female genitalia can generally be treated by performing a resection of the involved bowel (quality of evidence, very low; classification of recommendation, no specific recommendation).

- Level of agreement: strongly agree 5\%, agree 82\%, uncertain $5 \%$, disagree $8 \%$, strongly disagree $0 \%$
48. Surgery is preferred for enterovesical fistula. Medical treatment may be considered only for patients who have undergone multiple operations or are at high risk for short bowel syndrome (quality of evidence, very low; classification of recommendation, no specific recommendation).

- Level of agreement: strongly agree 9\%, agree 82\%, uncertain $2 \%$, disagree $7 \%$, strongly disagree $0 \%$

The treatment of female entrogenital fistulas in CD has not yet been established, but it has been reported that surgical treatment is superior to medical treatment. ${ }^{162}$ In 47 women with $\mathrm{CD}$ who had genital fistulas, the remission rates depended on medication type, which was reported as $0 \%$ antibiotics, 13\% immunomodulators, and $17 \%$ anti-TNF agents, and surgical treatment showed the remission rate of $22 \%$ after the first operation and $39 \%$ after the second operation. ${ }^{162}$ Meanwhile, infliximab showed moderate efficacy ( $45 \%$ fistula closure at week 14) in 25 patients with rectovaginal fistula among the ACCENT II subjects, and infliximab maintenance group had a longer duration of fistula closure than placebo group. ${ }^{163}$ In women with CD and enterogenital fistulas accompanied with severe symptoms in whom conservative treatment failed, surgery including fecal diversion is generally considered. Moreover, rectal resection may be necessary in patients with rectovaginal fistula with coexisting abscesses or complications. The surgical method should be carefully determined according to the individual patient's situations.

According to the report of 37 patients with CD and enterovesical fistulas, remission was achieved in $35.1 \%$ of patients after medical therapies including antibiotics, immunomodulators, corticosteroids, infliximab, or combination and these patients achieved remission could avoid surgery during the follow-up period of 4.7 years. ${ }^{164}$ Meanwhile, it has been reported that most of the patients with ileovesical fistulas who underwent surgery showed improved quality of life during long-term follow-up. ${ }^{165}$ Therefore, surgery is usually preferred for enterovesical fistulas; however, medical treatment can be considered in patients who already underwent multiple operations or are at high risk of short bowel syndrome.

\section{(3) Intra-abdominal abscess}

49. CD with intra-abdominal abscess should be treated with antibiotics, percutaneous or surgical drainage, and delayed bowel resection if necessary (quality of evidence, very low; classification of recommendation, no specific recommendation).

- Level of agreement: strongly agree 30\%, agree $67 \%$, uncertain $3 \%$, disagree $0 \%$, strongly disagree $0 \%$ 
50. If possible, percutaneous drainage under imaging guidance is performed (quality of evidence, very low; classification of recommendation, weak).

- Level of agreement: strongly agree 30\%, agree 65\%, uncertain 3\%, disagree 2\%, strongly disagree 0\%

51. For recurrent intra-abdominal abscesses after percutaneous drainage, or intra-abdominal abscess accompanied with associated fistula, surgery is considered (quality of evidence, very low; classification of recommendation, weak).

- Level of agreement: strongly agree 34\%, agree 64\%, uncertain $0 \%$, disagree $2 \%$, strongly disagree $0 \%$

If a patient with CD demonstrates fever, abdominal pain, or abdominal tenderness, an intra-abdominal abscess should be suspected, and in this case CT or ultrasound is helpful for diagnosis. Studies to date on the proper treatment of intra-abdominal abscess in patients with CD were mostly retrospective. Based on case reports and expert opinions, intra-abdominal abscesses should be treated with antibiotics, percutaneous or surgical drainage, and medical therapy for active disease should also be combined. ${ }^{13}$ Regarding drainage method, percutaneous drainage under CT or ultrasound guidance is preferred. The development of interventional procedures has resulted in percutaneous drainage being used as an alternative treatment to surgery or a bridging therapy before surgery. It has been reported that 50\% to $69 \%$ of patients who underwent percutaneous drainage could avoid surgery. ${ }^{166,167}$ In contrast, when an intra-abscess is accompanied with associated fistula, surgical treatment may be considered because of the higher possibility of percutaneous drainage failure or abscess relapse. ${ }^{168-170}$

Regarding the administration of immunomodulators after abscess development, it is unclear how long antibiotics should be used prior to starting immunomodulators and whether imaging studies are needed to confirm abscess disappearance prior to immunomodulator administration. However, experts suggest based on limited studies that if percutaneous drainage is successful in cases with spontaneous abscesses, proper anti-inflammatory treatment for inflamed bowel is beneficial for abscess healing and recurrence prevention; therefore, starting immunomodulators or anti-TNF agents as soon as possible is recommended. ${ }^{168}$ In contrast, in cases with intra-abdominal abscess subsequent to surgery, immunomodulators may not be needed immediately since the involved bowel segment is usually removed altogether in the surgery and immunomodulator therapy can increase the risk of sepsis. Thus, it is recommended that immunomodulators be administered after the abscess is completely cured in cases of postoperative intra-abdominal abscess. ${ }^{168}$

No studies to date have provided high evidence about whether subsequent bowel resection should be performed after percutaneous or surgical drainage. Previous studies tended to suggest bowel resection after abscess drainage. ${ }^{166,171,172}$ However, it was reported in recent years that nonsurgical treatment alone is effective. ${ }^{169,173,174}$ Delayed bowel resection is generally preferred when a patient has symptoms of bowel obstruction, ${ }^{171,172}$ whereas medical therapy alone may be considered if a patient has no symptoms of obstruction; however, this decision should be made based on each patient's clinical situation.

\section{SURGICAL TREATMENT OF CD}

52. Surgery is recommended for bowel perforation, uncontrolled bleeding, malignancy, and bowel obstruction or abscesses that unresponsive to medical treatment (quality of evidence, very low; classification of recommendation, weak).

- Level of agreement: strongly agree 78\%, agree 22\%, uncertain $0 \%$, disagree $0 \%$, strongly disagree $0 \%$

53. Surgery is considered for intractable stricture or fistula, active disease unresponsive to medical therapy, intractable extraintestinal complications such as pyoderma gangrenosum, and intractable perianal lesions (quality of evidence, very low; classification of recommendation, weak).

- Level of agreement: strongly agree 35\%, agree 65\%, uncertain 0\%, disagree 0\%, strongly disagree 0\%

54. When performing surgery for intractable stricture, only the involved bowel with stricture should be resected. Strictureplasty is considered when a short fibrous stricture of the small intestine is present or the length of the remaining small intestine is short (quality of evidence, very low; classification of recommendation, weak).

- Level of agreement: strongly agree 36\%, agree 64\%, uncertain $0 \%$, disagree $0 \%$, strongly disagree $0 \%$

A substantial number of patients with $\mathrm{CD}$ require surgery during the course of their illness. According to a recent metaanalysis, the 10-year risk of surgery after a diagnosis of CD was $46.6 \%$ (95\% CI, $37.7-57.7 \%) .{ }^{175}$ However, the surgery rate has tended to decrease over the past decade, which is thought to be because of the development of therapeutic agents such as biologics.

In most cases, surgery may not cure CD. However, since the ultimate goal of CD treatment is not to avoid surgery but to improve the patient's health and quality of life, ${ }^{25,26}$ surgery should be chosen if it can achieve this goal more quickly, 
safely, and effectively. ${ }^{176,177}$ In other words, ineffective medical treatment should not be continued to avoid surgery, and surgery should not be regarded as a last resort or a failure of medical treatment. ${ }^{26}$ In this regard, a physician must fully understand the benefits and risks of the surgery and choose it at the appropriate time. In addition, there is a need for indepth discussion with patients about the merits and drawbacks of both surgical and medical treatment.

The indications for surgery in patients with CD include bowel perforation, uncontrolled bleeding, persistent or recurrent bowel obstruction, despite medical treatment because of stricture, or malignant tumors. Surgery is also considered if there is no response to adequate medical treatment or if the medication cannot be continued because of side effects. ${ }^{11}$ Various evaluations should be performed to check for disease activity as well as the presence or absence of dysplasia, malignant tumor, and complications such as stricture, fistula, and abscess prior to surgery. Unlike immunomodulators or anti-TNF agents, preoperative systemic corticosteroid use (prednisolone $\geq 20 \mathrm{mg}$ for at least 2 weeks) increases the risk of postoperative infection complications. Therefore, systemic corticosteroids should be reduced before surgery whenever possible. ${ }^{178-180}$

In patients with $\mathrm{CD}$, postoperative relapse is irrelevant to the presence or absence of inflammation at both end of the resected bowel. ${ }^{181}$ Thus, for patients undergoing surgery because of intractable stricture, only the site-causing stricture is generally resected. Laparoscopic surgery recently became a safe and effective method of ileo-colonic resection in patients with CD and is preferred to laparotomy for simple resections. ${ }^{182}$ Strictureplasty may be considered to preserve the small intestine if fibrous stricture occurs over a short area of the small intestine or the remaining small intestine is short. The short- and long-term results of strictureplasty are reportedly equivalent to surgical resection. ${ }^{183}$ Traditional Heineke-Mikulicz strictureplasty is recommended for strictures $<10 \mathrm{~cm}$ long; however, for a wide range of diseases with long stricture, nontraditional strictureplasty such as side-toside strictureplasty may be attempted because of the possibility of postoperative short bowel syndrome. ${ }^{183}$

\section{POSTOPERATIVE TREATMENT OF CD}

CD cannot be cured by performing a surgery, and many patients with CD experience recurrence after surgery. Recurrence can be classified as endoscopic, radiologic, clinical, and surgical according to appearance pattern. Among them, endoscopic and radiologic recurrence refers cases in which a CD-related lesion can be observed on an endoscopic or radiologic examination after resection of all the grossly observed lesions, while clinical recurrence refers to a case in which symptoms of CD reappear. Finally, surgical recurrence is defined as the need for a second surgery because of medically refractory disease or complications. In patients with $\mathrm{CD}$, the cumulative reoperation rate after the first surgery is reportedly $16 \%$ to $43 \%$ at 5 years and $26 \%$ to $65 \%$ at 10 years. ${ }^{184,185}$

With regard to the monitoring of postoperative recurrence, most patients do not have symptoms until they develop into very severe lesions after surgery. ${ }^{186}$ Regarding postoperative recurrence, Rutgeerts et al. ${ }^{186}$ analyzed the long-term clinical recurrence and reoperation rates based on endoscopic lesions. They assessed the endoscopic lesions of the neoterminal ileum at the first year after curative resection and categorized them into five stages (i0, no lesions; il, $\leq 5$ aphthous lesions; i2, >5 aphthous lesions with normal mucosa; i3, diffuse aphthous ileitis with diffusely inflamed mucosa; and i4, diffuse inflammation with large ulcers, nodules, and/ or narrowing). When endoscopic recurrence was defined as an advanced stage $\geq i 2$, the clinical recurrence rates at 5 years were $30 \%$ in i2 and $50 \%$ to $100 \%$ in i 3 or i4, the latter of which were at high risk of reoperation. In contrast, in cases of i0 or il, the clinical recurrence rate at 10 years was $<10 \%$; thus, a favorable course was observed. ${ }^{186}$ These observations imply that endoscopic recurrence precedes clinical recurrence and that prognosis may be poor if endoscopic lesions are severe. As described above, colonoscopy is very useful for evaluating recurrence and predicting clinical course after surgery and may be helpful for determining the treatment strategy. A treatment strategy was recently developed for recommending proper drug therapy according to the postoperative endoscopic lesion, and studies on its usefulness are actively underway. ${ }^{187,188}$

Meanwhile, studies on the usefulness of cross-sectional imaging, such as CT, MRI, and contrast-enhanced ultrasonography have been reported as noninvasive methods for evaluating postoperative recurrence instead of colonoscopy. ${ }^{189-192}$ Other recent studies have shown that fecal calprotectin is useful for monitoring endoscopic recurrence in asymptomatic patients with $\mathrm{CD}$, and this test has been attracting attention as an alternative for endoscopy in the future. ${ }^{193,194}$ 


\section{Prevention of Postoperative Recurrence}

55. Smoking cessation is recommended in all patients after surgery (quality of evidence, low; classification of recommendation, weak).

- Level of agreement: strongly agree 87\%, agree 13\%, uncertain 0\%, disagree 0\%, strongly disagree 0\%.

56. 5-ASA may be helpful in maintaining remission of postoperative CD (quality of evidence, high; classification of recommendation, weak).

- Level of agreement: strongly agree 9\%, agree 80\%, uncertain 4\%, disagree 7\%, strongly disagree 0\%.

57. Thiopurine use is considered in patients at high risk of recurrence after surgery (quality of evidence, high; classification of recommendation, weak).

- Level of agreement: strongly agree 22\%, agree 69\%, uncertain 9\%, disagree 0\%, strongly disagree $0 \%$.

58. Anti-TNF therapy is considered for the prevention of postoperative recurrence in CD (quality of evidence, moderate; classification of recommendation, weak).

- Level of agreement: strongly agree 11\%, agree 71\%, uncertain $16 \%$, disagree $2 \%$, strongly disagree $0 \%$.

The major risk factors for postoperative recurrence in CD are smoking, previous bowel resection surgery, and fistulizing disease, and other factors such as the lack of treatment for recurrence prevention, perianal involvement, and extensive small bowel resection are also associated with recurrence. ${ }^{195}$ However, controversy persists about whether age at onset, sex, disease duration, involvement of resection margins, and surgery type is associated with recurrence. ${ }^{195}$

A meta-analysis of 834 postoperative CD patients showed that mesalamine significantly reduced postoperative clinical recurrence (RR, 0.80; 95\% CI, 0.70-0.92) compared with placebo or no treatment, and it was necessary to administer it for at least 10 weeks to exert its efficacy. ${ }^{196}$ A network meta-analysis of the effects of various drugs to prevent CD recurrence was recently reported. In this study, mesalamine significantly reduced clinical recurrence (RR, 0.60; 95\% CI, 0.37-0.88); however, it did not reduce the risk of endoscopic recurrence (RR, 0.67; 95\% CI, 0.39-1.08). ${ }^{197}$ Meanwhile, antibiotics reduced clinical recurrence (RR, 0.26; 95\% CI, 0.08-0.61) and endoscopic recurrence (RR, 0.41; 95\% CI, 0.15-0.92) compared to placebo. ${ }^{197}$ However, its use in clinical practice is limited because of the adverse effects and reduced efficacy during long-term use. In the same metaanalysis, the efficacy of immunomodulation was also analyzed. Thiopurine significantly reduced clinical recurrence (RR, 0.36; 95\% CI, 0.17-0.63) and endoscopic recurrence (RR, 0.33; 95\% CI, 0.13-0.68) compared to placebo. ${ }^{197}$ How- ever, thiopurine is not a completely safe drug, and although it has a low absolute risk, its long-term use may increase the patient's risk of lymphoma. ${ }^{198}$ In this regard, patients who are at high risk for recurrence may be preferentially considered for its administration. According to the meta-analysis (Supplementary Figs 2 and 3) performed in seven RCTs or nonrandomized comparative studies to analyze the efficacy of anti-TNF for preventing recurrence in postoperative CD, anti-TNF therapy significantly reduced endoscopic (RR, 0.21 ; 95\% CI, 0.14-0.32) and clinical recurrence (RR, 0.33; 95\% CI, 0.20-0.55) compared to placebo, mesalamine or immunomodulator therapies.

\section{CONCLUSIONS}

$\mathrm{CD}$ is a typical refractory disease with various clinical manifestations and long-term complications. In many clinical situations, physicians have difficulties in choosing the appropriate therapy for $\mathrm{CD}$ and often make clinical decisions according to the personal experience and subjective judgment. These guidelines have been designed to suggest an evidencebased therapeutic approach, and have been developed based on clinical data to date considering the domestic situation. We hope that these guidelines will help clinicians to choose the appropriate treatment for CD in a variety of clinical settings. Moreover, we wish problems such as over treatment, inadequate treatment, and delayed treatment of CD to be resolved through the dissemination of these guidelines.

\section{ACKNOWLEDGEMENTS}

We thank Dr. Edward Hyunseung Oh for his contribution to the editing of manuscript.

\section{REFERENCES}

1. Yang SK, Yun S, Kim JH, et al. Epidemiology of inflammatory bowel disease in the Songpa-Kangdong district, Seoul, Korea, 1986-2005: a KASID study. Inflamm Bowel Dis 2008;14:542-549.

2. Thia KT, Loftus EV Jr, Sandborn WJ, Yang SK. An update on the epidemiology of inflammatory bowel disease in Asia. Am J Gastroenterol 2008;103:3167-3182.

3. Ng WK, Wong SH, Ng SC. Changing epidemiological trends of inflammatory bowel disease in Asia. Intest Res 2016;14:111-119.

4. Ye BD, Yang SK, Shin SJ, et al. Guidelines for the management of Crohn's disease. Korean J Gastroenterol 2012;59:141-179.

5. Ye BD, Yang SK, Shin SJ, et al. Guidelines for the management of Crohn's disease. Intest Res 2012;10:22-66. 
6. D'Haens GR, Panaccione R, Higgins PD, et al. The London Position Statement of the World Congress of Gastroenterology on Biological Therapy for IBD with the European Crohn's and Colitis Organization: when to start, when to stop, which drug to choose, and how to predict response? Am J Gastroenterol 2011;106:199-212.

7. Orlando A, Armuzzi A, Papi C, et al. The Italian Society of Gastroenterology (SIGE) and the Italian Group for the Study of Inflammatory Bowel Disease (IG-IBD) Clinical Practice Guidelines: the use of tumor necrosis factor-alpha antagonist therapy in inflammatory bowel disease. Dig Liver Dis 2011;43:1-20.

8. Mowat C, Cole A, Windsor A, et al. Guidelines for the management of inflammatory bowel disease in adults. Gut 2011;60:571-607.

9. National Clinical Guideline Centre. National institute for health and clinical excellence: guidance. In: Crohn's disease: management in adults, children and young people. London: Royal College of Physicians (UK), 2012:1-398. Available at: https://www.ncbi.nlm.nih.gov/pubmedhealth/PMH0068978/. Accessed July 23, 2015.

10. Terdiman JP, Gruss CB, Heidelbaugh JJ, Sultan S, Falck-Ytter YT; AGA Institute Clinical Practice and Quality Management Committee. American Gastroenterological Association Institute guideline on the use of thiopurines, methotrexate, and anti-TNF-alpha biologic drugs for the induction and maintenance of remission in inflammatory Crohn's disease. Gastroenterology 2013;145:1459-1463.

11. Ueno F, Matsui T, Matsumoto T, et al. Evidence-based clinical practice guidelines for Crohn's disease, integrated with formal consensus of experts in Japan. J Gastroenterol 2013;48:31-72.

12. Gecse KB, Bemelman W, Kamm MA, et al. A global consensus on the classification, diagnosis and multidisciplinary treatment of perianal fistulising Crohn's disease. Gut 2014;63:1381-1392.

13. Ooi CJ, Makharia GK, Hilmi I, et al. Asia-Pacific consensus statements on Crohn's disease. Part 2: management. J Gastroenterol Hepatol 2016;31:56-68.

14. Balshem H, Helfand M, Schünemann HJ, et al. GRADE guidelines: 3. rating the quality of evidence. J Clin Epidemiol 2011;64:401-406.

15. Guyatt GH, Oxman AD, Vist GE, et al. GRADE: an emerging consensus on rating quality of evidence and strength of recommendations. BMJ 2008;336:924-926.

16. Lee KM, Kim YS, Seo GS, Kim TO, Yang SK; IBD Study Group of the Korean Association for the Study of Intestinal Diseases. Use of thiopurines in inflammatory bowel disease: a consensus statement by the Korean Association for the Study of Intestinal Diseases (KASID). Intest Res 2015;13:193-207.
17. Guyatt GH, Oxman AD, Kunz R, et al. Going from evidence to recommendations. BMJ 2008;336:1049-1051.

18. Regueiro M, Schraut W, Baidoo L, et al. Infliximab prevents Crohn's disease recurrence after ileal resection. Gastroenterology 2009;136:441-450.

19. Yamamoto T, Umegae S, Matsumoto K. Impact of infliximab therapy after early endoscopic recurrence following ileocolonic resection of Crohn's disease: a prospective pilot study. Inflamm Bowel Dis 2009;15:1460-1466.

20. Sorrentino D, Terrosu G, Paviotti A, et al. Early diagnosis and treatment of postoperative endoscopic recurrence of Crohn's disease: partial benefit by infliximab. A pilot study. Dig Dis Sci 2012;57:1341-1348.

21. Yoshida K, Fukunaga K, Ikeuchi H, et al. Scheduled infliximab monotherapy to prevent recurrence of Crohn's disease following ileocolic or ileal resection: a 3-year prospective randomized open trial. Inflamm Bowel Dis 2012;18:1617-1623.

22. Armuzzi A, Felice C, Papa A, et al. Prevention of postoperative recurrence with azathioprine or infliximab in patients with Crohn's disease: an open-label pilot study. J Crohns Colitis 2013;7:e623-e629. doi: 10.1016/j.crohns.2013.04.020.

23. Savarino E, Bodini G, Dulbecco P, et al. Adalimumab is more effective than azathioprine and mesalamine at preventing postoperative recurrence of Crohn's disease: a randomized controlled trial. Am J Gastroenterol 2013;108:1731-1742.

24. Regueiro M, Feagan BG, Zou B, et al. Infliximab reduces endoscopic, but not clinical, recurrence of Crohn's disease after ileocolonic resection. Gastroenterology 2016;150:1568-1578.

25. Sachar DB. Ten common errors in the management of inflammatory bowel disease. Inflamm Bowel Dis 2003;9:205-209.

26. Lichtenstein GR, Hanauer SB, Sandborn WJ; Practice Parameters Committee of American College of Gastroenterology. Management of Crohn's disease in adults. Am J Gastroenterol 2009;104:465-483.

27. Peyrin-Biroulet L, Sandborn W, Sands BE, et al. Selecting therapeutic targets in inflammatory bowel disease (STRIDE): determining therapeutic goals for treat-to-target. Am J Gastroenterol 2015;110:1324-1338.

28. Wolters FL, Russel MG, Stockbrügger RW. Systematic review: has disease outcome in Crohn's disease changed during the last four decades? Aliment Pharmacol Ther 2004;20:483-496.

29. Park SH, Yang SK, Park SK, et al. Long-term prognosis of Crohn's disease and its temporal change between 1981 and 2012: a hospital-based cohort study from Korea. Inflamm Bowel Dis 2014;20:488-494. 
30. Bernstein CN, Loftus EV Jr, Ng SC, Lakatos PL, Moum B; Epidemiology and Natural History Task Force of the International Organization for the Study of Inflammatory Bowel Disease (IOIBD). Hospitalisations and surgery in Crohn's disease. Gut 2012;61:622-629.

31. Peyrin-Biroulet L, Loftus EV Jr, Colombel JF, Sandborn WJ. The natural history of adult Crohn's disease in population-based cohorts. Am J Gastroenterol 2010;105:289-297.

32. Peyrin-Biroulet L, Loftus EV Jr, Colombel JF, Sandborn WJ. Long-term complications, extraintestinal manifestations, and mortality in adult Crohn's disease in population-based cohorts. Inflamm Bowel Dis 2011;17:471-478.

33. Beaugerie L, Seksik P, Nion-Larmurier I, Gendre JP, Cosnes J. Predictors of Crohn's disease. Gastroenterology 2006;130:650656.

34. Loly C, Belaiche J, Louis E. Predictors of severe Crohn's disease. Scand J Gastroenterol 2008;43:948-954.

35. Dignass A, Van Assche G, Lindsay JO, et al. The second European evidence-based consensus on the diagnosis and management of Crohn's disease: current management. J Crohns Colitis 2010;4:28-62.

36. Best WR, Becktel JM, Singleton JW, Kern F Jr. Development of a Crohn's disease activity index: National Cooperative Crohn's Disease Study. Gastroenterology 1976;70:439-444.

37. Harvey RF, Bradshaw JM. A simple index of Crohn's-disease activity. Lancet 1980;1:514

38. Van Assche G, Dignass A, Panes J, et al. The second European evidence-based consensus on the diagnosis and management of Crohn's disease: definitions and diagnosis. J Crohns Colitis 2010;4:7-27.

39. Sandborn WJ, Feagan BG, Hanauer SB, et al. A review of activity indices and efficacy endpoints for clinical trials of medical therapy in adults with Crohn's disease. Gastroenterology 2002;122:512-530.

40. Rutgeerts P, D'Haens G, Targan S, et al. Efficacy and safety of retreatment with anti-tumor necrosis factor antibody (infliximab) to maintain remission in Crohn's disease. Gastroenterology 1999;117:761-769.

41. Hanauer SB, Feagan BG, Lichtenstein GR, et al. Maintenance infliximab for Crohn's disease: the ACCENT I randomised trial. Lancet 2002;359:1541-1549.

42. Vermeire S, Schreiber S, Sandborn WJ, Dubois C, Rutgeerts P. Correlation between the Crohn's disease activity and HarveyBradshaw indices in assessing Crohn's disease severity. Clin Gastroenterol Hepatol 2010;8:357-363.

43. Stange EF, Travis SP, Vermeire S, et al. European evidence based consensus on the diagnosis and management of Crohn's disease: definitions and diagnosis. Gut 2006;55 Suppl 1:i1-i15.
44. Gasche C, Scholmerich J, Brynskov J, et al. A simple classification of Crohn's disease: report of the Working Party for the World Congresses of Gastroenterology, Vienna 1998. Inflamm Bowel Dis 2000;6:8-15.

45. Satsangi J, Silverberg MS, Vermeire S, Colombel JF. The Montreal classification of inflammatory bowel disease: controversies, consensus, and implications. Gut 2006;55:749-753.

46. Louis E, Collard A, Oger AF, Degroote E, Aboul Nasr El Yafi FA, Belaiche J. Behaviour of Crohn's disease according to the Vienna classification: changing pattern over the course of the disease. Gut 2001;49:777-782.

47. Cosnes J, Cattan S, Blain A, et al. Long-term evolution of disease behavior of Crohn's disease. Inflamm Bowel Dis 2002;8:244-250.

48. Lim WC, Hanauer S. Aminosalicylates for induction of remission or response in Crohn's disease. Cochrane Database Syst Rev 2010;(12):CD008870. doi: 10.1002/14651858.CD008870.

49. Malchow H, Ewe K, Brandes JW, et al. European Cooperative Crohn's Disease Study (ECCDS): results of drug treatment. Gastroenterology 1984;86:249-266.

50. Anthonisen P, Barany F, Folkenborg O, et al. The clinical effect of salazosulphapyridine (Salazopyrin r) in Crohn's disease: a controlled double-blind study. Scand J Gastroenterol 1974:9:549-554.

51. Van Hees PA, Van Lier HJ, Van Elteren PH, et al. Effect of sulphasalazine in patients with active Crohn's disease: a controlled double-blind study. Gut 1981;22:404-409.

52. Singleton JW, Hanauer SB, Gitnick GL, et al. Mesalamine capsules for the treatment of active Crohn's disease: results of a 16-week trial. Pentasa Crohn's Disease Study Group. Gastroenterology 1993;104:1293-1301.

53. Prantera C, Cottone M, Pallone F, et al. Mesalamine in the treatment of mild to moderate active Crohn's ileitis: results of a randomized, multicenter trial. Gastroenterology 1999;116:521526.

54. Hanauer SB, Stromberg U. Oral Pentasa in the treatment of active Crohn's disease: a meta-analysis of double-blind, placebocontrolled trials. Clin Gastroenterol Hepatol 2004;2:379-388.

55. Seow CH, Benchimol EI, Steinhart AH, Griffiths AM, Otley AR. Budesonide for Crohn's disease. Expert Opin Drug Metab Toxicol 2009;5:971-979.

56. Seow CH, Benchimol EI, Griffiths AM, Otley AR, Steinhart AH. Budesonide for induction of remission in Crohn's disease. Cochrane Database Syst Rev 2008;(3):CD000296. doi: 10.1002/14651858.CD000296.pub3. 
57. Campieri M, Ferguson A, Doe W, Persson T, Nilsson LG. Oral budesonide is as effective as oral prednisolone in active Crohn's disease: the Global Budesonide Study Group. Gut 1997;41:209-214.

58. Gross V, Andus T, Caesar I, et al. Oral pH-modified release budesonide versus 6-methylprednisolone in active Crohn's disease: German/Austrian Budesonide Study Group. Eur J Gastroenterol Hepatol 1996;8:905-909.

59. Benchimol EI, Seow CH, Steinhart AH, Griffiths AM. Traditional corticosteroids for induction of remission in Crohn's disease. Cochrane Database Syst Rev 2008;(2):CD006792. doi: 10.1002/14651858.CD006792.pub2.

60. Summers RW, Switz DM, Sessions JT Jr, et al. National Cooperative Crohn's Disease Study: results of drug treatment. Gastroenterology 1979;77:847-869.

61. Steinhart AH, Ewe K, Griffiths AM, Modigliani R, Thomsen OO. Corticosteroids for maintenance of remission in Crohn's disease. Cochrane Database Syst Rev 2003;(4):CD000301. doi: 10.1002/14651858.CD000301.

62. Targan SR, Hanauer SB, van Deventer SJ, et al. A short-term study of chimeric monoclonal antibody cA2 to tumor necrosis factor alpha for Crohn's disease: Crohn's Disease cA2 Study Group. N Engl J Med 1997;337:1029-1035.

63. Hanauer SB, Sandborn WJ, Rutgeerts P, et al. Human antitumor necrosis factor monoclonal antibody (adalimumab) in Crohn's disease: the CLASSIC-I trial. Gastroenterology 2006;130:323-333.

64. Schreiber S, Rutgeerts P, Fedorak RN, et al. A randomized, placebo-controlled trial of certolizumab pegol (CDP870) for treatment of Crohn's disease. Gastroenterology 2005;129:807818.

65. Sandborn WJ, Feagan BG, Stoinov S, et al. Certolizumab pegol for the treatment of Crohn's disease. N Engl J Med 2007;357:228-238.

66. Sandborn WJ, Schreiber S, Feagan BG, et al. Certolizumab pegol for active Crohn's disease: a placebo-controlled, randomized trial. Clin Gastroenterol Hepatol 2011;9:670-678.

67. Pearson DC, May GR, Fick GH, Sutherland LR. Azathioprine and 6-mercaptopurine in Crohn disease: a meta-analysis. Ann Intern Med 1995;123:132-142.

68. Chande N, Tsoulis DJ, MacDonald JK. Azathioprine or 6-mercaptopurine for induction of remission in Crohn's disease. Cochrane Database Syst Rev 2013;(4):CD000545. doi: 10.1002/14651858.CD000545.pub4.

69. Colombel JF, Sandborn WJ, Reinisch W, et al. Infliximab, azathioprine, or combination therapy for Crohn's disease. N Engl J Med 2010;362:1383-1395.
70. Kopylov U, Al-Taweel T, Yaghoobi M, et al. Adalimumab monotherapy versus combination therapy with immunomodulators in patients with Crohn's disease: a systematic review and meta-analysis. J Crohns Colitis 2014;8:1632-1641.

71. Goodsell DS. The molecular perspective: methotrexate. Oncologist 1999;4:340-341.

72. Rajagopalan PT, Zhang Z, McCourt L, Dwyer M, Benkovic SJ, Hammes GG. Interaction of dihydrofolate reductase with methotrexate: ensemble and single-molecule kinetics. Proc Natl Acad Sci U S A 2002;99:13481-13486.

73. Cronstein BN. The mechanism of action of methotrexate. Rheum Dis Clin North Am 1997;23:739-755.

74. Feagan BG, Rochon J, Fedorak RN, et al. Methotrexate for the treatment of Crohn's disease: the North American Crohn's Study Group Investigators. N Engl J Med 1995;332:292-297.

75. Arora S, Katkov W, Cooley J, et al. Methotrexate in Crohn's disease: results of a randomized, double-blind, placebo-controlled trial. Hepatogastroenterology 1999;46:1724-1729.

76. Oren R, Moshkowitz M, Odes S, et al. Methotrexate in chronic active Crohn's disease: a double-blind, randomized, Israeli multicenter trial. Am J Gastroenterol 1997;92:2203-2209.

77. Ben-Horin S, Kopylov U, Chowers Y. Optimizing anti-TNF treatments in inflammatory bowel disease. Autoimmun Rev 2014;13:24-30.

78. Siegel CA, Melmed GY. Predicting response to Anti-TNF agents for the treatment of Crohn's disease. Therap Adv Gastroenterol 2009;2:245-251.

79. Colombel JF, Sandborn WJ, Rutgeerts P, et al. Adalimumab for maintenance of clinical response and remission in patients with Crohn's disease: the CHARM trial. Gastroenterology 2007;132:52-65.

80. Vermeire S, Louis E, Carbonez A, et al. Demographic and clinical parameters influencing the short-term outcome of anti-tumor necrosis factor (infliximab) treatment in Crohn's disease. Am J Gastroenterol 2002;97:2357-2363.

81. Parsi MA, Achkar JP, Richardson S, et al. Predictors of response to infliximab in patients with Crohn's disease. Gastroenterology 2002;123:707-713.

82. Ho GT, Smith L, Aitken S, et al. The use of adalimumab in the management of refractory Crohn's disease. Aliment Pharmacol Ther 2008;27:308-315.

83. Swaminath A, Ullman T, Rosen M, Mayer L, Lichtiger S, Abreu MT. Early clinical experience with adalimumab in treatment of inflammatory bowel disease with infliximab-treated and naive patients. Aliment Pharmacol Ther 2009;29:273-278.

84. Ben-Horin S, Chowers Y. Review article: loss of response to anti-TNF treatments in Crohn's disease. Aliment Pharmacol Ther 2011;33:987-995. 
85. Papamichael K, Gils A, Rutgeerts P, et al. Role for therapeutic drug monitoring during induction therapy with TNF antagonists in IBD: evolution in the definition and management of primary nonresponse. Inflamm Bowel Dis 2015;21:182-197.

86. Karmiris K, Paintaud G, Noman M, et al. Influence of trough serum levels and immunogenicity on long-term outcome of adalimumab therapy in Crohn's disease. Gastroenterology 2009;137:1628-1640.

87. Steenholdt C, Bendtzen K, Brynskov J, Ainsworth MA. Optimizing treatment with TNF inhibitors in inflammatory bowel disease by monitoring drug levels and antidrug antibodies. Inflamm Bowel Dis 2016;22:1999-2015.

88. Sands BE, Feagan BG, Rutgeerts P, et al. Effects of vedolizumab induction therapy for patients with Crohn's disease in whom tumor necrosis factor antagonist treatment failed. Gastroenterology 2014;147:618-627.e3.

89. Feagan BG, Sandborn WJ, Gasink C, et al. Ustekinumab as Induction and Maintenance Therapy for Crohn's Disease. N Engl J Med 2016;375:1946-1960.

90. Sandborn WJ, Rutgeerts P, Enns R, et al. Adalimumab induction therapy for Crohn disease previously treated with infliximab: a randomized trial. Ann Intern Med 2007;146:829-838.

91. Akobeng AK, Gardener E. Oral 5-aminosalicylic acid for maintenance of medically-induced remission in Crohn's disease. Cochrane Database Syst Rev 2005;(1):CD003715. doi: 10.1002/14651858.CD003715.pub2.

92. Steinhart AH, Forbes A, Mills EC, Rodgers-Gray BS, Travis SP. Systematic review: the potential influence of mesalazine formulation on maintenance of remission in Crohn's disease. Aliment Pharmacol Ther 2007;25:1389-1399.

93. Ford AC, Bernstein CN, Khan KJ, et al. Glucocorticosteroid therapy in inflammatory bowel disease: systematic review and meta-analysis. Am J Gastroenterol 2011;106:590-599.

94. Candy S, Wright J, Gerber M, Adams G, Gerig M, Goodman R. A controlled double blind study of azathioprine in the management of Crohn's disease. Gut 1995;37:674-678.

95. Dassopoulos T, Sultan S, Falck-Ytter YT, Inadomi JM, Hanauer SB. American Gastroenterological Association Institute technical review on the use of thiopurines, methotrexate, and antiTNF-alpha biologic drugs for the induction and maintenance of remission in inflammatory Crohn's disease. Gastroenterology 2013;145:1464-1478.

96. Behm BW, Bickston SJ. Tumor necrosis factor-alpha antibody for maintenance of remission in Crohn's disease. Cochrane Database Syst Rev 2008;(1):CD006893. doi: 10.1002/14651858. CD006893.
97. Feagan BG, Panaccione R, Sandborn WJ, et al. Effects of adalimumab therapy on incidence of hospitalization and surgery in Crohn's disease: results from the CHARM study. Gastroenterology 2008;135:1493-1499.

98. Schreiber S, Khaliq-Kareemi M, Lawrance IC, et al. Maintenance therapy with certolizumab pegol for Crohn's disease. N Engl J Med 2007;357:239-250.

99. Rutgeerts P, Feagan BG, Lichtenstein GR, et al. Comparison of scheduled and episodic treatment strategies of infliximab in Crohn's disease. Gastroenterology 2004;126:402-413.

100. Maser EA, Villela R, Silverberg MS, Greenberg GR. Association of trough serum infliximab to clinical outcome after scheduled maintenance treatment for Crohn's disease. Clin Gastroenterol Hepatol 2006;4:1248-1254.

101. Rutgeerts P, Diamond RH, Bala M, et al. Scheduled maintenance treatment with infliximab is superior to episodic treatment for the healing of mucosal ulceration associated with Crohn's disease. Gastrointest Endosc 2006;63:433-442.

102. Van Assche G, Magdelaine-Beuzelin C, D'Haens G, et al. Withdrawal of immunosuppression in Crohn's disease treated with scheduled infliximab maintenance: a randomized trial. Gastroenterology 2008;134:1861-1868.

103. Sokol H, Seksik P, Carrat F, et al. Usefulness of co-treatment with immunomodulators in patients with inflammatory bowel disease treated with scheduled infliximab maintenance therapy. Gut 2010;59:1363-1368.

104. Viazis N, Koukouratos T, Anastasiou J, et al. Azathioprine discontinuation earlier than 6 months in Crohn's disease patients started on anti-TNF therapy is associated with loss of response and the need for anti-TNF dose escalation. Eur J Gastroenterol Hepatol 2015;27:436-441.

105. Kierkus J, Iwanczak B, Wegner A, et al. Monotherapy with infliximab versus combination therapy in the maintenance of clinical remission in children with moderate to severe Crohn disease. J Pediatr Gastroenterol Nutr 2015;60:580-585.

106. Jones JL, Kaplan GG, Peyrin-Biroulet L, et al. Effects of concomitant immunomodulator therapy on efficacy and safety of anti-tumor necrosis factor therapy for Crohn's disease: a metaanalysis of placebo-controlled trials. Clin Gastroenterol Hepatol 2015;13:2233-2240.e2.

107. Feagan BG, Fedorak RN, Irvine EJ, et al. A comparison of methotrexate with placebo for the maintenance of remission in Crohn's disease: North American Crohn's Study Group Investigators. N Engl J Med 2000;342:1627-1632.

108. Patel V, Wang Y, MacDonald JK, McDonald JW, Chande N. Methotrexate for maintenance of remission in Crohn's disease. Cochrane Database Syst Rev 2014;(8):CD006884. doi: 10.1002/14651858.CD006884.pub3. 
109. Nathan DM, Iser JH, Gibson PR. A single center experience of methotrexate in the treatment of Crohn's disease and ulcerative colitis: a case for subcutaneous administration. J Gastroenterol Hepatol 2008;23:954-958.

110. Mayberry JF, Lobo A, Ford AC, Thomas A. NICE clinical guideline (CG152): the management of Crohn's disease in adults, children and young people. Aliment Pharmacol Ther 2013;37:195-203.

111. Regueiro M, Siemanowski B, Kip KE, Plevy S. Infliximab dose intensification in Crohn's disease. Inflamm Bowel Dis 2007;13:1093-1099.

112. Sandborn WJ, Colombel JF, Schreiber S, et al. Dosage adjustment during long-term adalimumab treatment for Crohn's disease: clinical efficacy and pharmacoeconomics. Inflamm Bowel Dis 2011;17:141-151.

113. Sandborn WJ, Feagan BG, Rutgeerts P, et al. Vedolizumab as induction and maintenance therapy for Crohn's disease. N Engl J Med 2013;369:711-721.

114. Hanauer SB. Inflammatory bowel disease: epidemiology, pathogenesis, and therapeutic opportunities. Inflamm Bowel Dis 2006;12 Suppl 1:S3-S9.

115. Stocchi L, Milsom JW, Fazio VW. Long-term outcomes of laparoscopic versus open ileocolic resection for Crohn's disease: follow-up of a prospective randomized trial. Surgery 2008;144:622-627.

116. Eshuis EJ, Slors JF, Stokkers PC, et al. Long-term outcomes following laparoscopically assisted versus open ileocolic resection for Crohn's disease. Br J Surg 2010;97:563-568.

117. Eshuis EJ, Stokkers PC, Bemelman WA. Decision-making in ileocecal Crohn's disease management: surgery versus pharmacotherapy. Expert Rev Gastroenterol Hepatol 2010;4:181189.

118. Wagtmans MJ, Verspaget HW, Lamers CB, van Hogezand RA. Clinical aspects of Crohn's disease of the upper gastrointestinal tract: a comparison with distal Crohn's disease. Am J Gastroenterol 1997;92:1467-1471.

119. Mottet C, Juillerat P, Pittet V, et al. Upper gastrointestinal Crohn's disease. Digestion 2007;76:136-140.

120. Miehsler W, Püspök A, Oberhuber T, Vogelsang H. Impact of different therapeutic regimens on the outcome of patients with Crohn's disease of the upper gastrointestinal tract. Inflamm Bowel Dis 2001;7:99-105.

121. Hanauer SB, Sandborn W; Practice Parameters Committee of the American College of Gastroenterology. Management of Crohn's disease in adults. Am J Gastroenterol 2001;96:635-643.
122. Travis SP, Stange EF, Lémann M, et al. European evidence based consensus on the diagnosis and management of Crohn's disease: current management. Gut 2006;55 Suppl 1:i16-i35.

123. Matsui T, Hatakeyama S, Ikeda K, Yao T, Takenaka K, Sakurai T. Long-term outcome of endoscopic balloon dilation in obstructive gastroduodenal Crohn's disease. Endoscopy 1997;29:640645.

124. Matsui T, Ikeda K, Tsuda S, et al. Long-term outcome of endoscopic balloon dilation in obstructive gastrointestinal Crohn's disease: a prospective long-term study. Diagn Ther Endosc 2000;6:67-75.

125. Singh VV, Draganov P, Valentine J. Efficacy and safety of endoscopic balloon dilation of symptomatic upper and lower gastrointestinal Crohn's disease strictures. J Clin Gastroenterol 2005;39:284-290.

126. Tonelli F, Alemanno G, Bellucci F, Focardi A, Sturiale A, Giudici F. Symptomatic duodenal Crohn's disease: is strictureplasty the right choice? J Crohns Colitis 2013;7:791-796.

127. Vader JP, Froehlich F, Juillerat P, et al. Appropriate treatment for Crohn's disease. Methodology and summary results of a multidisciplinary international expert panel approach: EPACT. Digestion 2006;73:237-248.

128. Thomas-Gibson S, Brooker JC, Hayward CM, Shah SG, Williams CB, Saunders BP. Colonoscopic balloon dilation of Crohn's strictures: a review of long-term outcomes. Eur J Gastroenterol Hepatol 2003;15:485-488.

129. Lichtenstein GR, Olson A, Travers S, et al. Factors associated with the development of intestinal strictures or obstructions in patients with Crohn's disease. Am J Gastroenterol 2006;101:1030-1038.

130. Condino G, Calabrese E, Zorzi F, et al. Anti-TNF-alpha treatments and obstructive symptoms in Crohn's disease: a prospective study. Dig Liver Dis 2013;45:258-262.

131. Present DH, Rutgeerts $P$, Targan S, et al. Infliximab for the treatment of fistulas in patients with Crohn's disease. N Engl J Med 1999;340:1398-1405.

132. Irvine EJ. Usual therapy improves perianal Crohn's disease as measured by a new disease activity index: McMaster IBD Study Group. J Clin Gastroenterol 1995;20:27-32.

133. Sandborn WJ, Fazio VW, Feagan BG, Hanauer SB; American Gastroenterological Association Clinical Practice Committee. AGA technical review on perianal Crohn's disease. Gastroenterology 2003;125:1508-1530.

134. Schwartz DA, Loftus EV Jr, Tremaine WJ, et al. The natural history of fistulizing Crohn's disease in Olmsted County, Minnesota. Gastroenterology 2002;122:875-880. 
135. Cheon JH, Kim YS, Ye BD, et al. Crohn's Disease Clinical Network and Cohort (CONNECT) study: the first step toward nationwide multicenter research of Crohn's disease in Korea. Intest Res 2014;12:173-175.

136. Jung YS, Park DI, Ye BD, et al. Long-term clinical outcomes of urban versus rural environment in Korean patients with Crohn's disease: results from the CONNECT study. J Crohns Colitis 2015;9:246-251.

137. Kim B, Cheon JH, Moon HJ, et al. Crohn's disease prognosis and early immunomodulator therapy: results from the CONNECT study. J Gastroenterol Hepatol 2016;31:126-132.

138. Bernstein LH, Frank MS, Brandt LJ, Boley SJ. Healing of perineal Crohn's disease with metronidazole. Gastroenterology 1980;79:599

139. Brandt LJ, Bernstein LH, Boley SJ, Frank MS. Metronidazole therapy for perineal Crohn's disease: a follow-up study. Gastroenterology 1982;83:383-387.

140. Jakobovits J, Schuster MM. Metronidazole therapy for Crohn's disease and associated fistulae. Am J Gastroenterol 1984;79:533-540.

141. Thia KT, Mahadevan U, Feagan BG, et al. Ciprofloxacin or metronidazole for the treatment of perianal fistulas in patients with Crohn's disease: a randomized, double-blind, placebocontrolled pilot study. Inflamm Bowel Dis 2009;15:17-24.

142. Levien DH, Surrell J, Mazier WP. Surgical treatment of anorectal fistula in patients with Crohn's disease. Surg Gynecol Obstet 1989;169:133-136.

143. Schwartz DA, Maltz BE. Treatment of fistulizing inflammatory bowel disease. Gastroenterol Clin North Am 2009;38:595-610.

144. Dejaco C, Harrer M, Waldhoer T, Miehsler W, Vogelsang H, Reinisch W. Antibiotics and azathioprine for the treatment of perianal fistulas in Crohn's disease. Aliment Pharmacol Ther 2003;18:1113-1120.

145. Egan LJ, Sandborn WJ, Tremaine WJ. Clinical outcome following treatment of refractory inflammatory and fistulizing Crohn's disease with intravenous cyclosporine. Am J Gastroenterol 1998;93:442-448.

146. Present DH, Lichtiger S. Efficacy of cyclosporine in treatment of fistula of Crohn's disease. Dig Dis Sci 1994;39:374-380.

147. Sandborn WJ, Present DH, Isaacs KL, et al. Tacrolimus for the treatment of fistulas in patients with Crohn's disease: a randomized, placebo-controlled trial. Gastroenterology 2003;125:380-388.

148. Mahadevan U, Marion JF, Present DH. Fistula response to methotrexate in Crohn's disease: a case series. Aliment Pharmacol Ther 2003;18:1003-1008.
149. Sands BE, Anderson FH, Bernstein CN, et al. Infliximab maintenance therapy for fistulizing Crohn's disease. N Engl J Med 2004;350:876-885.

150. Colombel JF, Schwartz DA, Sandborn WJ, et al. Adalimumab for the treatment of fistulas in patients with Crohn's disease. Gut 2009;58:940-948.

151. Lichtiger S, Binion DG, Wolf DC, et al. The CHOICE trial: adalimumab demonstrates safety, fistula healing, improved quality of life and increased work productivity in patients with Crohn's disease who failed prior infliximab therapy. Aliment Pharmacol Ther 2010;32:1228-1239.

152. Echarri A, Castro J, Barreiro M, Carpio D, Pereira S, Lorenzo A. Evaluation of adalimumab therapy in multidisciplinary strategy for perianal Crohn's disease patients with infliximab failure J Crohns Colitis 2010;4:654-660.

153. Dewint P, Hansen BE, Verhey E, et al. Adalimumab combined with ciprofloxacin is superior to adalimumab monotherapy in perianal fistula closure in Crohn's disease: a randomised, double-blind, placebo controlled trial (ADAFI). Gut 2014;63:292299.

154. Yassin NA, Askari A, Warusavitarne J, et al. Systematic review: the combined surgical and medical treatment of fistulising perianal Crohn's disease. Aliment Pharmacol Ther 2014;40:741-749.

155. Regueiro M. The role of endoscopy in the evaluation of fistulizing Crohn's disease. Gastrointest Endosc Clin N Am 2002;12:621-633.

156. Bell SJ, Williams AB, Wiesel P, Wilkinson K, Cohen RC, Kamm MA. The clinical course of fistulating Crohn's disease. Aliment Pharmacol Ther 2003;17:1145-1151.

157. Guillem JG, Roberts PL, Murray JJ, Coller JA, Veidenheimer MC, Schoetz DJ Jr. Factors predictive of persistent or recurrent Crohn's disease in excluded rectal segments. Dis Colon Rectum 1992;35:768-772.

158. Rehg KL, Sanchez JE, Krieger BR, Marcet JE. Fecal diversion in perirectal fistulizing Crohn's disease is an underutilized and potentially temporary means of successful treatment. Am Surg 2009;75:715-718.

159. Panés J, García-Olmo D, Van Assche G, et al. Expanded allogeneic adipose-derived mesenchymal stem cells (Cx601) for complex perianal fistulas in Crohn's disease: a phase 3 randomised, double-blind controlled trial. Lancet 2016;388:12811290.

160. Lee WY, Park KJ, Cho YB, et al. Autologous adipose tissuederived stem cells treatment demonstrated favorable and sustainable therapeutic effect for Crohn's fistula. Stem Cells 2013;31:2575-2581. 
161. Amiot A, Setakhr V, Seksik P, et al. Long-term outcome of enterocutaneous fistula in patients with Crohn's disease treated with anti-TNF therapy: a cohort study from the GETAID. Am J Gastroenterol 2014;109:1443-1449.

162. de la Poza G, López-Sanroman A, Taxonera C, et al. Genital fistulas in female Crohn's disease patients: clinical characteristics and response to therapy. J Crohns Colitis 2012;6:276-280.

163. Sands BE, Blank MA, Patel K, van Deventer SJ; ACCENT II Study. Long-term treatment of rectovaginal fistulas in Crohn's disease: response to infliximab in the ACCENT II Study. Clin Gastroenterol Hepatol 2004;2:912-920.

164. Zhang W, Zhu W, Li Y, et al. The respective role of medical and surgical therapy for enterovesical fistula in Crohn's disease. J Clin Gastroenterol 2014;48:708-711.

165. Hastings JW, Garg M, Lynn ET, Divino CM. Surgical repair of ileovesical fistulas: long-term complications, quality of life, and patient satisfaction. Am Surg 2014;80:1207-1211.

166. Gervais DA, Hahn PF, O'Neill MJ, Mueller PR. Percutaneous abscess drainage in Crohn disease: technical success and short- and long-term outcomes during 14 years. Radiology 2002;222:645-651.

167. Gutierrez A, Lee H, Sands BE. Outcome of surgical versus percutaneous drainage of abdominal and pelvic abscesses in Crohn's disease. Am J Gastroenterol 2006;101:2283-2289.

168. Feagins LA, Holubar SD, Kane SV, Spechler SJ. Current strategies in the management of intra-abdominal abscesses in Crohn's disease. Clin Gastroenterol Hepatol 2011;9:842-850.

169. Lee H, Kim YH, Kim JH, et al. Nonsurgical treatment of abdominal or pelvic abscess in consecutive patients with Crohn's disease. Dig Liver Dis 2006;38:659-664.

170. Sahai A, Bélair M, Gianfelice D, Coté S, Gratton J, Lahaie R. Percutaneous drainage of intra-abdominal abscesses in Crohn's disease: short and long-term outcome. Am J Gastroenterol 1997;92:275-278.

171. Garcia JC, Persky SE, Bonis PA, Topazian M. Abscesses in Crohn's disease: outcome of medical versus surgical treatment. J Clin Gastroenterol 2001;32:409-412.

172. Yamaguchi A, Matsui T, Sakurai T, et al. The clinical characteristics and outcome of intraabdominal abscess in Crohn's disease. J Gastroenterol 2004;39:441-448.

173. Kim DH, Cheon JH, Moon CM, et al. Clinical efficacy of nonsurgical treatment of Crohn's disease-related intraabdominal abscess. Korean J Gastroenterol 2009;53:29-35.

174. Nguyen DL, Sandborn WJ, Loftus EV Jr, et al. Similar outcomes of surgical and medical treatment of intra-abdominal abscesses in patients with Crohn's disease. Clin Gastroenterol Hepatol 2012;10:400-404.
175. Frolkis AD, Dykeman J, Negrón ME, et al. Risk of surgery for inflammatory bowel diseases has decreased over time: a systematic review and meta-analysis of population-based studies. Gastroenterology 2013;145:996-1006.

176. McLeod RS. Surgery for inflammatory bowel diseases. Dig Dis 2003;21:168-179.

177. Thirlby RC, Land JC, Fenster LF, Lonborg R. Effect of surgery on health-related quality of life in patients with inflammatory bowel disease: a prospective study. Arch Surg 1998;133:826832.

178. Aberra FN, Lewis JD, Hass D, Rombeau JL, Osborne B, Lichtenstein GR. Corticosteroids and immunomodulators: postoperative infectious complication risk in inflammatory bowel disease patients. Gastroenterology 2003;125:320-327.

179. Marchal L, D'Haens G, Van Assche G, et al. The risk of postoperative complications associated with infliximab therapy for Crohn's disease: a controlled cohort study. Aliment Pharmacol Ther 2004;19:749-754.

180. Van Assche G, Dignass A, Reinisch W, et al. The second European evidence-based consensus on the diagnosis and management of Crohn's disease: special situations. J Crohns Colitis 2010;4:63-101.

181. Post S, Herfarth C, Böhm E, et al. The impact of disease pattern, surgical management, and individual surgeons on the risk for relaparotomy for recurrent Crohn's disease. Ann Surg 1996;223:253-260.

182. Tan JJ, Tjandra JJ. Laparoscopic surgery for Crohn's disease: a meta-analysis. Dis Colon Rectum 2007;50:576-585.

183. Bharadwaj S, Fleshner P, Shen B. Therapeutic armamentarium for stricturing Crohn's disease: medical versus endoscopic versus surgical approaches. Inflamm Bowel Dis 2015;21:21942213.

184. Williams JG, Wong WD, Rothenberger DA, Goldberg SM. Recurrence of Crohn's disease after resection. Br J Surg 1991;78:10-19

185. Ye BD, Yang SK, Cho YK, et al. Clinical features and long-term prognosis of Crohn's disease in Korea. Scand J Gastroenterol 2010;45:1178-1185.

186. Rutgeerts P, Geboes K, Vantrappen G, Beyls J, Kerremans R, Hiele M. Predictability of the postoperative course of Crohn's disease. Gastroenterology 1990;99:956-963.

187. De Cruz P, Kamm MA, Hamilton AL, et al. Crohn's disease management after intestinal resection: a randomised trial. Lancet 2015;385:1406-1417.

188. Yamamoto T, Watanabe T. Strategies for the prevention of postoperative recurrence of Crohn's disease. Colorectal Dis 2013;15:1471-1480. 
189. Koilakou S, Sailer J, Peloschek P, et al. Endoscopy and MR enteroclysis: equivalent tools in predicting clinical recurrence in patients with Crohn's disease after ileocolic resection. Inflamm Bowel Dis 2010;16:198-203.

190. Minordi LM, Vecchioli A, Poloni G, Guidi L, De Vitis I, Bonomo L. Enteroclysis CT and PEG-CT in patients with previous small-bowel surgical resection for Crohn's disease: CT findings and correlation with endoscopy. Eur Radiol 2009;19:2432 2440.

191. Paredes JM, Ripollés T, Cortés X, et al. Contrast-enhanced ultrasonography: usefulness in the assessment of postoperative recurrence of Crohn's disease. J Crohns Colitis 2013;7:192-201.

192. Papay P, Ignjatovic A, Karmiris K, et al. Optimising monitoring in the management of Crohn's disease: a physician's perspective. J Crohns Colitis 2013;7:653-669.

193. Boschetti G, Laidet M, Moussata D, et al. Levels of fecal calprotectin are associated with the severity of postoperative endoscopic recurrence in asymptomatic patients with Crohn's disease. Am J Gastroenterol 2015;110:865-872.
194. Wright EK, Kamm MA, De Cruz P, et al. Measurement of fecal calprotectin improves monitoring and detection of recurrence of Crohn's disease after surgery. Gastroenterology 2015;148:938-947.

195. Fornaro R, Caratto E, Caratto M, et al. Post-operative recurrence in Crohn's disease. Critical analysis of potential risk factors: an update. Surgeon 2015;13:330-347.

196. Ford AC, Khan KJ, Talley NJ, Moayyedi P. 5-aminosalicylates prevent relapse of Crohn's disease after surgically induced remission: systematic review and meta-analysis. Am J Gastroenterol 2011;106:413-420.

197. Singh S, Garg SK, Pardi DS, Wang Z, Murad MH, Loftus EV Jr. Comparative efficacy of pharmacologic interventions in preventing relapse of Crohn's disease after surgery: a systematic review and network meta-analysis. Gastroenterology 2015;148:64-76.e2.

198. Kotlyar DS, Lewis JD, Beaugerie L, et al. Risk of lymphoma in patients with inflammatory bowel disease treated with azathioprine and 6-mercaptopurine: a meta-analysis. Clin Gastroenterol Hepatol 2015;13:847-858.e4. 
See “Second Korean guidelines for the management of Crohn's disease" on page 38.

Supplementary Table 1. Details of the Search Strategy

\begin{tabular}{|c|c|}
\hline Search engine & Search detail \\
\hline PubMed & 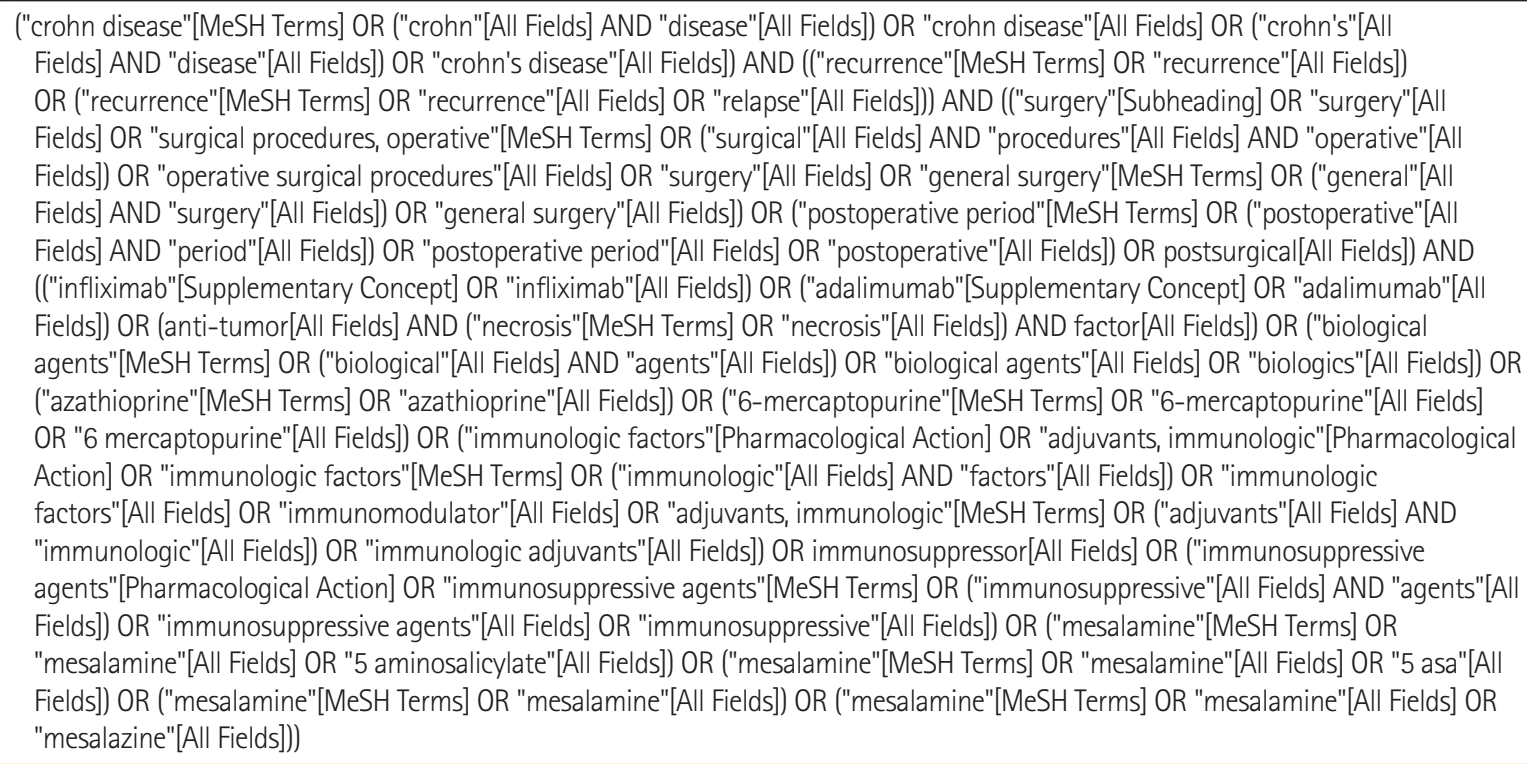 \\
\hline EMBASE & $\begin{array}{l}\text { ('crohn disease') AND ('adalimumab' OR 'immunomodulating agent' OR 'infliximab' OR 'methotrexate'/ OR 'monoclonal antibody'/ OR } \\
\text { 'tumor necrosis factor alpha' AND ('case control study OR 'clinical article' OR 'clinical trial' OR 'cohort analysis' OR 'comparative study' } \\
\text { OR 'controlled clinical trial'om/de OR 'controlled study' OR 'cross-sectional study' OR 'double blind procedure' OR ' OR 'major clinical } \\
\text { study' OR 'medical record review' OR 'multicenter study' OR 'observational study' OR 'open study' OR 'pilot study' OR 'prospective } \\
\text { study' OR 'randomized controlled trial' OR 'retrospective study') }\end{array}$ \\
\hline
\end{tabular}


See "Second Korean guidelines for the management of Crohn's disease" on page 38.

Supplementary Table 2. Summary of the Included Studies

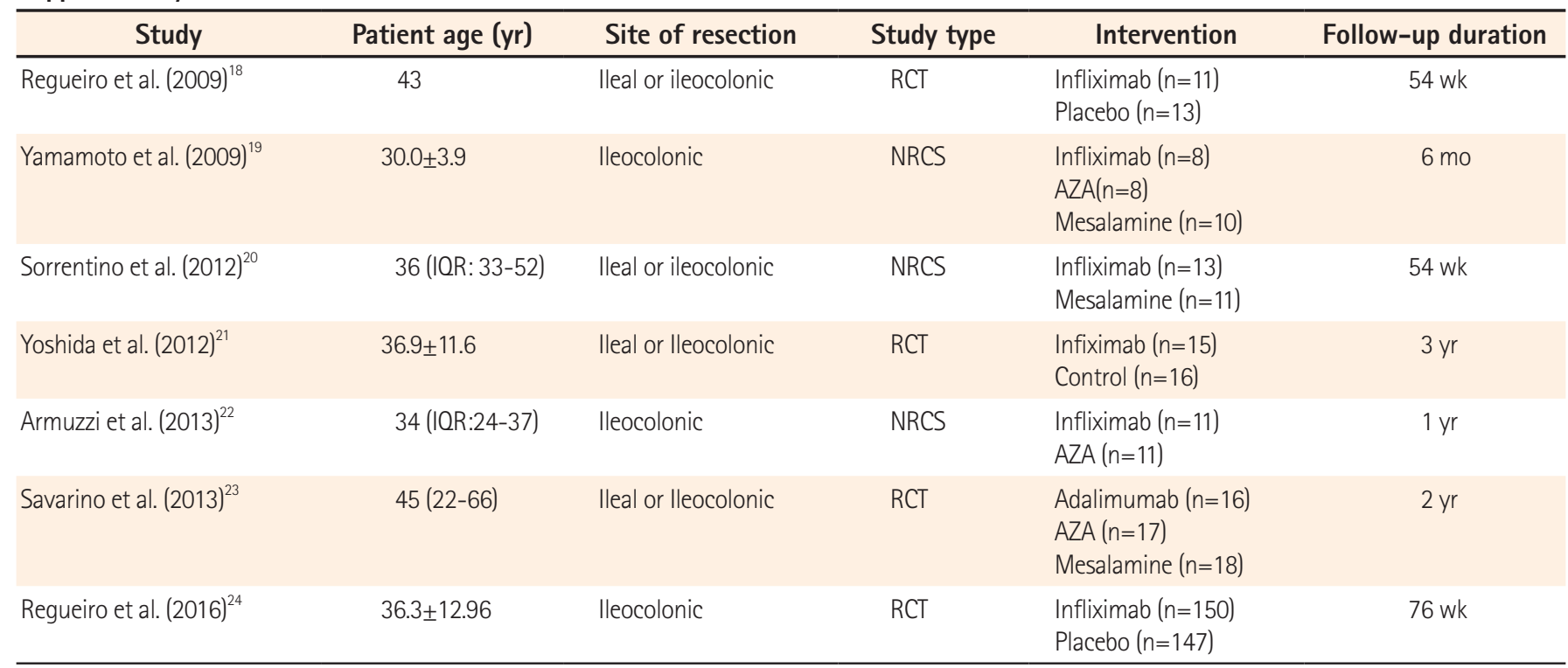

IQR, interquartile range; $R C T$, randomized controlled trial; NRCS, nonrandomized comparative study; AZA, azathioprine. 
See "Second Korean guidelines for the management of Crohn's disease" on page 38.

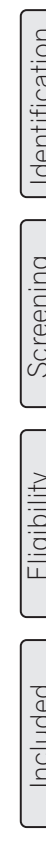

5,889 Records identified through database searching (PubMed, EMBASE)

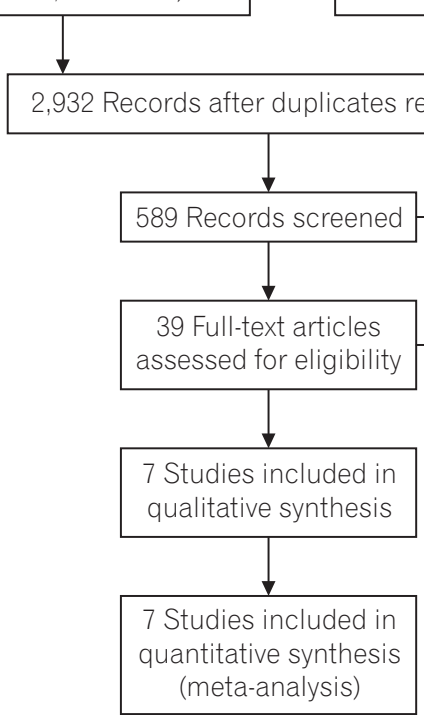

0 Additional records identified through other sources
32 Full-text articles excluded, with reasons 9 Not relevant subject

8 Systematic review and meta-analysis

6 Review publication

4 Editorial, letter etc.

3 Case series/no control group

1 Pediatric patients

1 Costeffective analysis
Supplementary Fig. 1. Flow diagram of the study selection process. 
See "Second Korean guidelines for the management of Crohn's disease" on page 38.

A

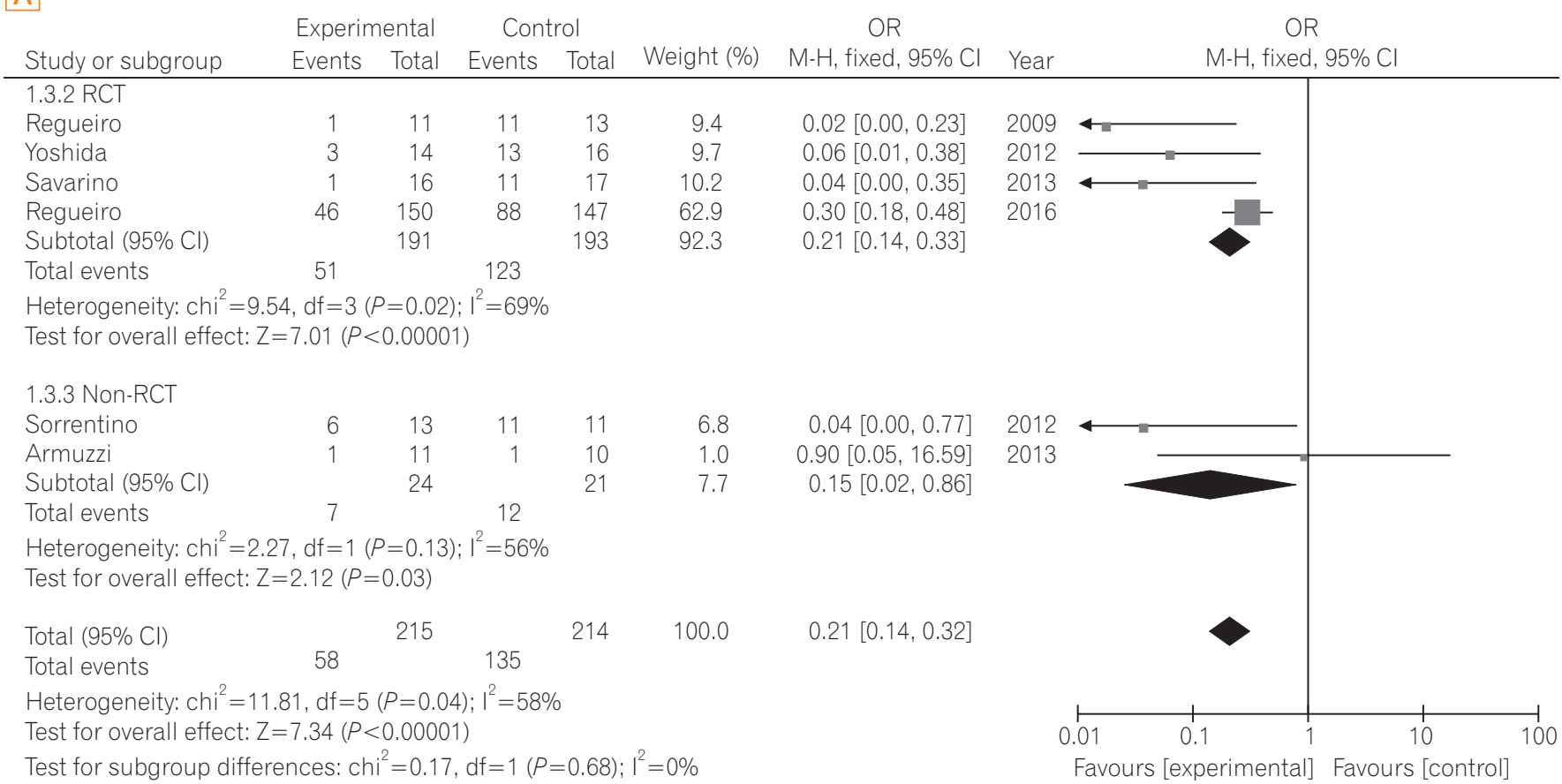

B

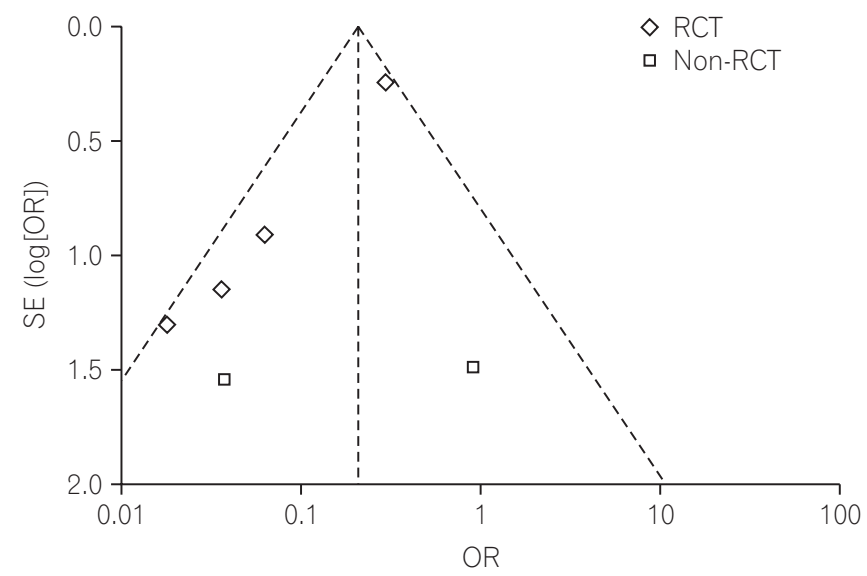

Supplementary Fig. 2. (A) Forest plots of fixed-effects comparison of anti-tumor necrosis factor- $\alpha$ versus control for postoperative endoscopic recurrence. (B) Funnel plot for publication bias among studies with endoscopic recurrence. RCT, randomized controlled trial; SE, standard error. 
See "Second Korean guidelines for the management of Crohn's disease" on page 38.

A

\begin{tabular}{lrrrrrc} 
& \multicolumn{2}{c}{ Experimental } & \multicolumn{2}{c}{ Control } & OR \\
Study or subgroup & Events & Total & Events & Total & Weight (\%) & M-H, fixed, 95\% C \\
\hline 2.3.2 RCT & 0 & 10 & 5 & 13 & 8.4 & $0.07[0.00,1.53]$ \\
Regueiro & 19 & 150 & 30 & 147 & 48.4 & $0.57[0.30,1.06]$ \\
Regueiro & 2 & 16 & 9 & 18 & 13.5 & $0.14[0.02,0.82]$ \\
Savarino & 0 & 8 & 7 & 10 & 11.7 & $0.03[0.00,0.62]$ \\
Yamamoto & & 184 & & 188 & 82.0 & $0.37[0.21,0.63]$ \\
Subtotal (95\% Cl) & 21 & & 51 & & &
\end{tabular}

Heterogeneity: $\mathrm{chi}^{2}=6.67, \mathrm{df}=3(P=0.08) ;\left.\right|^{2}=55 \%$

Test for overall effect: $Z=3.61(P=0.0003)$

\subsubsection{Non-RCT}

Armuzzi

Sorrentino

Yamamoto

Subtotal $(95 \% \mathrm{Cl})$

Total events

$\begin{array}{rr}1 & 10 \\ 0 & 13 \\ 0 & 8 \\ & 31\end{array}$

1
2
7

Heterogeneity: $\mathrm{chi}^{2}=2.95, \mathrm{df}=2(P=0.23): 1^{2}=32 \%$

Test for overall effect: $Z=2.40(P=0.02)$

$\begin{array}{lllllll}\text { Total }(95 \% \mathrm{Cl}) & 22 & 215 & & 220 & 100.0 & 0.33[0.20,0.55] \\ \text { Total events } & 22 & & 61 & & & \end{array}$

Heterogeneity: chi $^{2}=10.49, \mathrm{df}=6(P=0.11) ;\left.\right|^{2}=43 \%$

Test for overall effect: $Z=4.29(P<0.0001)$

Test for subgroup differences: chi $^{2}=1.13, \mathrm{df}=1(P=0.29) ; I^{2}=11.8 \%$

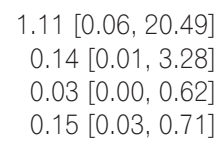

$0.15[0.03,0.71]$

$$
18.0
$$

$\begin{array}{rr}1.6 & 1.11[0.06,20.49] \\ 4.7 & 0.14[0.01,3.28] \\ 11.7 & 0.03[0.00,0.62] \\ 18.0 & 0.15[0.03,0.71]\end{array}$

M-H, fixed, 95\%

OR

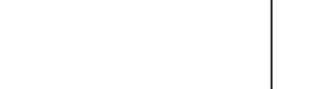

B

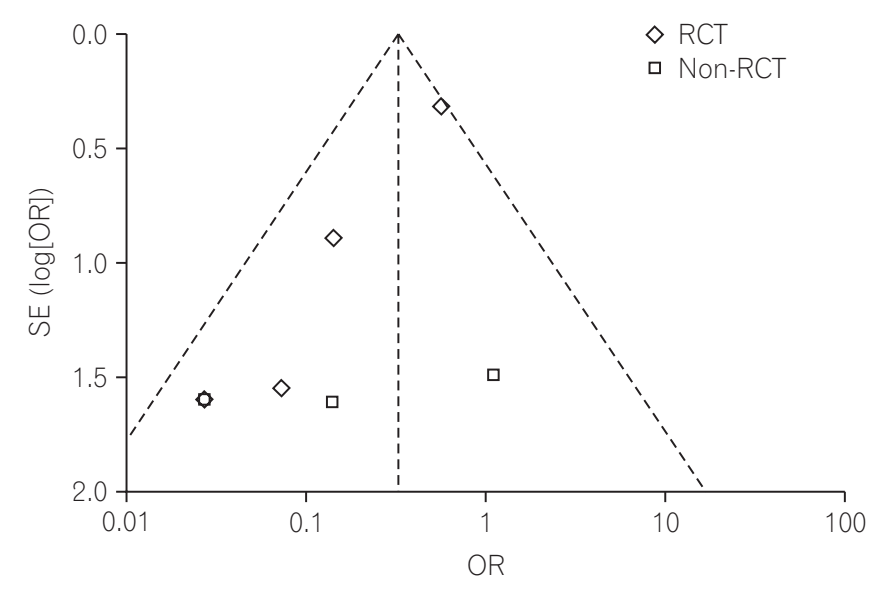

Supplementary Fig. 3. (A) Forest plots of fixed-effects comparison of anti-tumor necrosis factor- $\alpha$ versus control for postoperative clinical recurrence. (B) Funnel plot for publication bias among studies with clinical recurrence. RCT, randomized controlled trial; SE, standard error. 